\title{
Guided ionization waves: The physics of repeatability
}

\author{
XinPei Lu ${ }^{1,2, a)}$ and Kostya (Ken) Ostrikov ${ }^{3,4}$ \\ ${ }^{1}$ State Key Laboratory of Advanced Electromagnetic Engineering and Technology, Huazhong University \\ of Science and Technology, Wuhan, Hubei 430030, People's Republic of China \\ ${ }^{2}$ IFSA Collaborative Innovation Center, Shanghai Jiao Tong University, Shanghai 200240, \\ People's Republic of China \\ ${ }^{3}$ School of Chemistry, Physics and Mechanical Engineering, Queensland University of Technology, Brisbane, \\ Queensland 4000, Australia \\ ${ }^{4}$ CSIRO-QUT Joint Sustainable Processes and Devices Laboratory, P. O. Box 218, Lindfield, NSW 2070, \\ Australia
}

(Received 29 March 2018; accepted 22 May 2018; published online 24 July 2018)

\begin{abstract}
Guided ionization waves, or plasma streamers, are increasingly important for many applications in spanning materials processing and biomedicine. The highly reproducible, repeatable behavior of the most puzzling kind of the streamers-plasma bullets is highly attractive as it promises a high degree of control in many applications. However, despite a dozen years since the discovery of this phenomenon, the exact reasons for such behavior still remain essentially unclear. To understand the dynamics of the guided ionization wave (plasma bullet), a large number of works have been carried out and many interesting results have been reported. Here, we critically examine the available results and generalize the physical mechanisms of the guided ionization waves, which are of particular interest to practical applications of atmospheric-pressure plasma discharges, in general. The critical examination of the fundamental principles will show that, in order to propagate in a repeatable-mode, the plasma bullet must propagate in a channel with a high seed electron density (HSED), which is on the order of $10^{9} \mathrm{~cm}^{-3}$. This review concludes that to distinguish guided ionization waves from traditional positive streamer discharges, it is most appropriate to describe an atmospheric-pressure discharge featuring a plasma bullet behavior as an HSED discharge. When the HSED condition is met, the dynamics of a plasma plume appears to be repeatable. On the contrary, it propagates in an unrepeatable mode and emerges more like a positive streamer discharge when the HSED condition is not satisfied. According to this theory, the transition of the propagation mode of the plasma bullet between the repeatable mode and the stochastic mode can be well explained. Besides by controlling the seed electron density around the transition region between the HSED discharge and the traditional positive streamer, this knowledge will help in better understanding of the positive streamer discharges in air, in cases relevant to practical applications of such plasma discharges in materials processing technologies, industrial chemistry, nanotechnology, and health care. Published by AIP Publishing. https://doi.org/10.1063/1.5031445
\end{abstract}

\section{TABLE OF CONTENTS}

I. INTRODUCTION $\ldots \ldots \ldots \ldots \ldots \ldots \ldots \ldots \ldots$

II. TOWNSEND THEORY $\ldots \ldots \ldots \ldots \ldots \ldots \ldots . \ldots \ldots$

III. STREAMER THEORY ............... 4

A. The branching behavior ................ 4

B. Effect of photoionization ........... 5

C. Effect of seed electron density ......... 7

D. Effect of frequency of the applied voltage . . 9

E. Effect of gas pressure .............. 10

F. Effect of adding radiative gas . ......... 10

G. Preliminary summary ........... 11

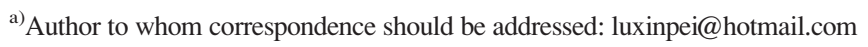

IV. GUIDED IONIZATION WAVE: HIGH SEED ELECTRON DENSITY (HSED) DISCHARGE .

A. Plasma bullet propagation: Repeatable and

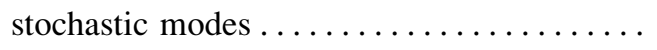

B. Propagation mode of the first discharge

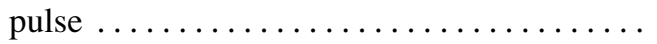

C. Minimum seed electron density for repeatable mode at different gas pressures .. 13

D. Photoionization for plasma bullet........ 15

V. PHYSICAL MECHANISMS ............ 16

A. Minimum SED for repeatable propagation mode in pure helium plasma........... 16

B. Minimum SED for repeatable propagation

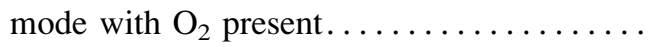
1. SED due to $\mathrm{O}_{2}{ }^{-}$detachment .......... 17 2. SED due to photoionization........ 17 
C. Required minimum SED for repeatable

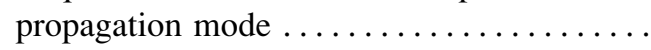

VI. FUTURE CHALLENGES AND OPPORTUNITIES . .................

A. Minimum seed electron density for air streamer propagating in repeatable mode...

B. Stabilization period of repeatable propagation mode of plasma bullet .......

C. Detachment rate of $\mathrm{O}_{2}^{-} \ldots \ldots \ldots \ldots \ldots$

D. Photoionization models $\ldots \ldots \ldots \ldots \ldots \ldots$

E. Streamer simulation models...........

F. New phenomena in plasma plumes........ VII. CONCLUSION ...................

\section{INTRODUCTION}

Nonequilibrium atmospheric pressure plasma jets $(\mathrm{N}-$ APPJs) have attracted a lot of attention in the past decade due to several emerging applications such as plasma medicine and materials processing. ${ }^{1-10}$ To fulfill the requirement of various applications, many different types of N-APPJ devices have been developed. In the meantime, studies on the dynamics of N-APPJs, i.e., the guided ionization waves, or the commonly called "plasma bullet," have found that the guided ionization wave propagates at a speed of $10^{3}-10^{6} \mathrm{~m} / \mathrm{s}$, which is similar to the propagation speed of positive streamers. ${ }^{11-20}$

However, there are some distinct differences between guided ionization waves and positive streamers. First, positive steamer discharges normally have lots of branches as shown in Fig. 1. The number and the diameter of the branches depend on many parameters including the gas component, the gas pressure, and the amplitude of the applied voltage. On the other hand, guided ionization waves propagate along a straight line, aligned with the flow path of the working gas (typically $\mathrm{He}$ or Ar) as shown in Fig. 2, and propagate the same distance after the same delay time as shown in Fig. 3.

Second, the repeatability of the discharges is different between these discharge types. A positive streamer does not propagate the same distance for the same delay time after the voltage applied among different shots. This could be due to two aspects, i.e., (1) the ignition jitter of the streamer and (2) the variation of the propagation velocity of the streamer from pulse to pulse. Regarding the ignition jitter, it varies from tens of ns when pure $\mathrm{N}_{2}$ is used to microseconds when a small amount of $\mathrm{O}_{2}$ is added. ${ }^{24}$ Regarding the variation of propagation velocity of a streamer from pulse to pulse, until now, almost all measurements on the propagation velocity of streamers are based on swarm data. This uncertainty arises because of the multiple branches and the un-reproducible characteristics of streamers. Indeed, the streamers do not propagate along the same path for different shots, whereas a typical variation of the propagation speed is about $20 \%-50 \% .^{25}$

Recent advances in high-speed multi-frame intensified charge-coupled device (ICCD) imaging enable accurate measurements of the propagation velocity of a streamer for a single shot. ${ }^{25}$ The obtained propagation speed of the streamer has a fluctuation as high as $50 \%$. On the other hand, guided ionization waves show excellent repeatability; ${ }^{26}$ the fluctuation of the ignition moment of the plasma plume is generally less than just a few nanoseconds and the variation of the propagation speed is less than several percent. In short, guided ionization waves always propagate the same distance after the same delay time.

Third, a streamer does not even propagate along the same path among consecutive discharge pulses as shown in Fig. 1. The images show that subsequent streamers do not follow the same path. Only some channels (outside the crowded central area) are highlighted in cyan, yellow, or white, while most of the channels are in red, green, or blue. Regarding the guided ionization wave, it always propagates along the same path aligned with the flow of the working gas.

Importantly, there are several new phenomena intrinsic to guided ionization waves which do not appear in streamers. For example, when a pulsed direct current (DC) voltage pulse is used to drive a plasma plume, the plasma plume appears a continued bright plume in surrounding air (similar to Fig. 2) when the pulse width is much shorter than its pulse duration. However, when the pulse width is close to the pulse duration, in other words, when the pulse off time is only a few microseconds or several hundred nanoseconds, the plasma plume appears in two or three segments depending on the pulse off time, as can be seen in Fig. 4. The area next to the nozzle could be either much darker or brighter than the area located several millimeters away from the nozzle. Another example is the snake-like propagation behavior of the plasma bullet as shown in Fig. $5 .^{27}$ Interestingly, snake-
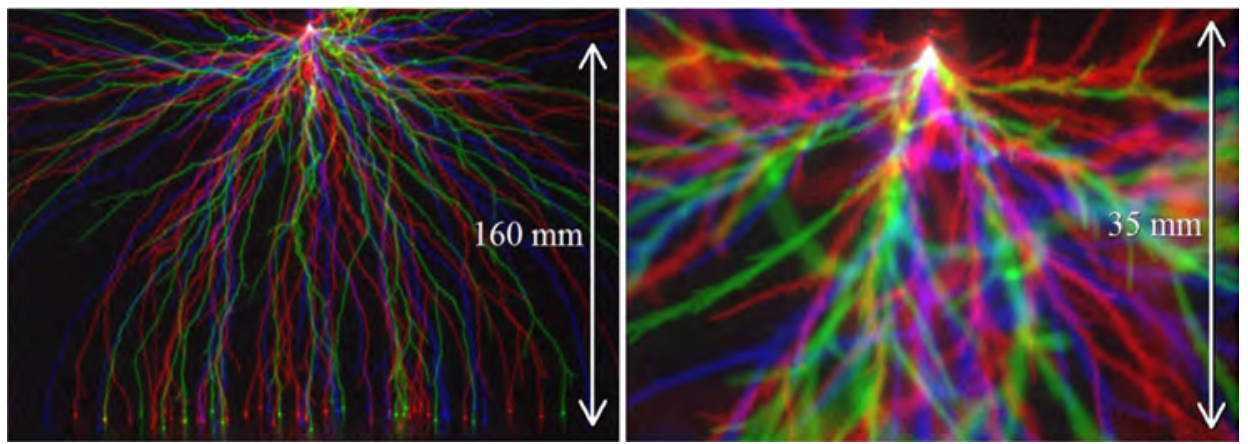

FIG. 1. Three consecutive images at $10 \mathrm{~Hz}$ repetition rate coloured in red, green, and blue, respectively, and overlaid. Left: overview, right: zoomed. The repetitive streamer paths are highlighted in yellow (red + green), cyan (green + blue), or white $($ red + green + blue), while single streamer paths are highlighted in red, green, and blue. All images are acquired in 200 mbar N ${ }_{2}{ }^{21}$ Reproduced with permission from Nijdam et al., J. Phys. D: Appl. Phys. 44, 455201 (2011). Copyright 2011 Institute of Physics. 


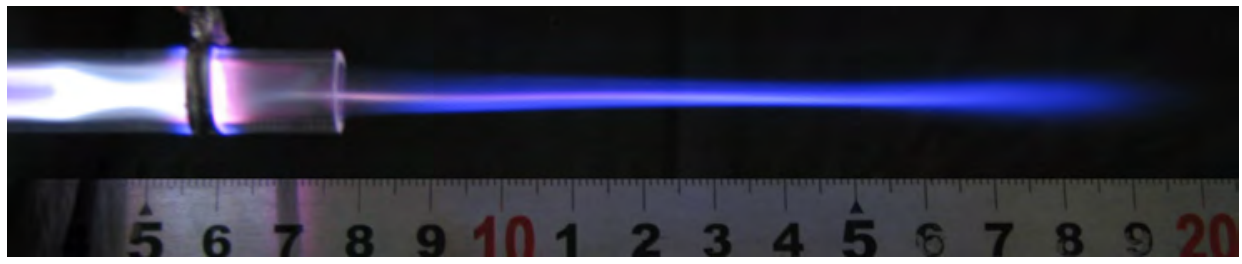

FIG. 2. Photograph of a typical plasma plume of guided ionization waves. ${ }^{22}$ Reproduced with permission from Appl. Phys. Lett. 92, 081502 (2008). Copyright 2008 AIP Publishing LLC.

like streamers appear both in the upstream and downstream plasma plumes. This unusual behavior is very different from the straight-line propagation reported previously.

This review aims to help better understand and interpret the repeatable dynamics of the guided ionization waves and the associated phenomena. The well-known Townsend theory is described briefly in Sec. II. Then, the traditional streamer theory including the branching behavior, the effects of photoionization, seed electron density (SED) $n_{\text {seed }}$, frequency of the applied voltage, and adding of radiative gas on the ignition and propagation dynamics are examined in Sec. III. In Sec. IV, state-of the-art research on the propagation repeatability of guided ionization waves (plasma bullets) is discussed. The associated physical phenomena include repeatable and stochastic modes of the plasma bullet, the propagation mode of the very first discharge pulse, minimum $\mathrm{n}_{\text {seed }}$ for a repeatable mode to exist at different gas pressures, and the effect of photoionization on the propagation of plasma bullets. Critical discussion on the reported modeling and experimental works to establish the requirements for the plasma plume to propagate in a repeatable mode is presented in Sec. V. Future challenges and opportunities are highlighted in Sec. VI. Finally, concluding remarks are given in Sec. VII.

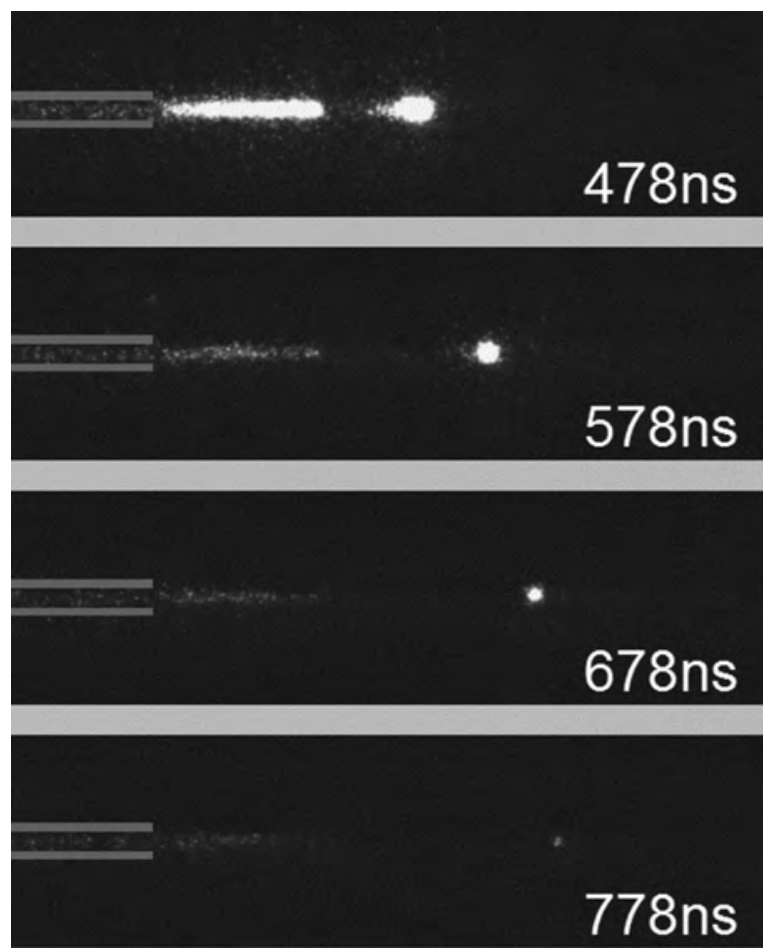

FIG. 3. High-speed photographs of repetitive plasma bullets propagating along the straight line. The exposure time is $5 \mathrm{~ns}$. Voltage: $8 \mathrm{kV}$, pulse width: $998 \mu \mathrm{s}$, frequency: $1 \mathrm{kHz}$, and helium flow rate: $1 \mathrm{l} / \mathrm{min} .{ }^{23}$ Reproduced with permission from Xian et al., Sci. Rep. 3, 1599 (2013). Copyright 2013 Springer Nature LLC.

\section{TOWNSEND THEORY}

Due to remarkable differences between plasma bullets and traditional streamer discharges discussed above, it is critical to know the fundamental nature of the plasma bullet and traditional streamer. The fundamental understanding of plasma discharges takes its roots from Townsend's discharge theory ${ }^{28}$ proposed more than a century ago.

The Townsend theory is based on the assumption that an electron, which might have originated due to ionization caused by cosmic radiation, drifts towards the anode under electric field. If the electric field is strong enough, the free electron gains sufficient energy to liberate a further electron when it collides with a molecule. The two free electrons then travel towards the anode and gain sufficient energy from the electric field. Electron impact ionization takes place upon subsequent collisions, and then newly produced electrons cause the same effect. This process is effectively a chain reaction of electron generation; it depends on the free electrons gaining sufficient energy between collisions to sustain the avalanche. The total number of electrons reaching the anode is equal to the number of collisions plus the single initiating free electron. Assuming the number of ionizing collisions per unit length is $\alpha$, which is known as the first Townsend coefficient, then the total number of free electrons after the travel distance $x$ reaching the anode is $\exp (\alpha x){ }^{29}$

In the meantime, the number of positive ions generated during the avalanche is $\exp (\alpha x)-1$. When a positive ion impacts the cathode, it may result in the emission of an electron. Denoting the average number of electrons released from a surface by an incident positive ion as $\gamma$, the condition for a self-sustainable discharge requires that $\gamma[\exp (\alpha x)-1]$ $>1$. Townsend theory predicts the experimental results very well only when the electric field distribution is uniform, the

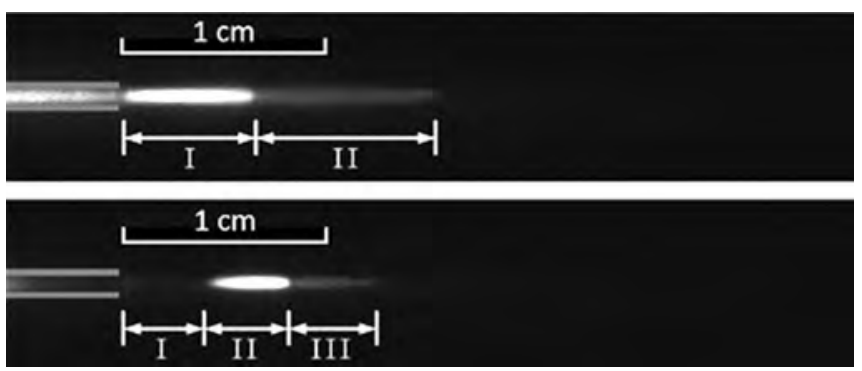

FIG. 4. Photographs of two distinctive plasma plume types generated using different sequences of DC voltage pulses: the plume on top has bright (I) and $\operatorname{dim}$ (II) areas (pulse width: $998 \mu \mathrm{s}$ ), whereas the plume at the bottom features dark (I), bright (II), and $\operatorname{dim}$ (III) areas (pulse width: $999.1 \mu \mathrm{s}$ ). All the other parameters for the two cases are the same, which are voltage: $8 \mathrm{kV}$, frequency: $1 \mathrm{kHz}$, and helium flow rate: $11 / \mathrm{min} .{ }^{23}$ Reproduced with permission from Xian et al., Sci. Rep. 3, 1599 (2013). Copyright 2013 Springer Nature LLC. 


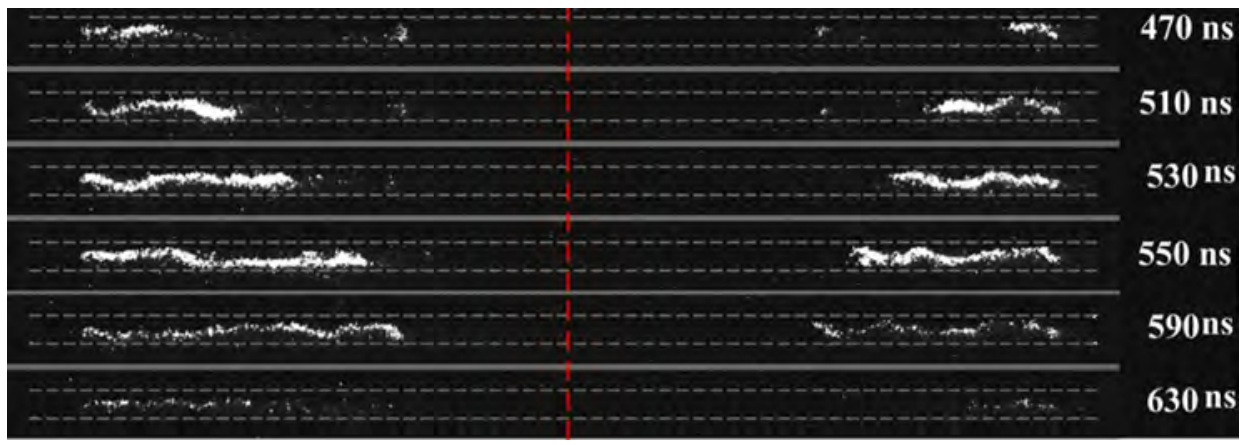

FIG. 5. Snake-like propagation of repetitive streamers in both upstream and downstream areas imaged by ICCD. The exposure time is fixed at $4 \mathrm{~ns}$. Voltage: $8 \mathrm{kV}$, pulse width: $400 \mathrm{~ns}$, frequency: $9 \mathrm{kHz}$, and argon gas flow rate: 51/min. ${ }^{27}$ Reproduced with permission from Phys. Plasmas 20, 023503 (2013). Copyright 2013 AIP Publishing LLC. gas pressure is low, and the gap distance is small, leading to the theory validity condition $p d<200$ Torr $\cdot \mathrm{cm}$.

However, this theory is inconsistent with the observations for discharges that have a larger gap $d$ or are sustained at a higher gas pressure $p$. In other words, when $p d$ $>200$ Torr $\cdot \mathrm{cm}$, the breakdown time appears to be much shorter than that predicted by the Townsend theory. Under such conditions, positive ions need more time to reach the cathode. In addition, according to the Townsend theory, the breakdown depends on the ability of the cathode to release electrons upon ion impact. However, experimental results show that this is not true for most of the experimental observations. Besides, the Townsend theory does not consider the effect of the space charge left behind the electron avalanche generated earlier. In many instances, the concentration of positive ions can reach very appreciable values that could significantly distort the initial electric field. This might lead to the locally augmented electron energies and ionization, where the resulting ionization rate $\alpha$ could be well above its value in a static and uniform electric field. Furthermore, in many instances, the spark channel at high values of $p d$ was found to be both branched and zig-zagged, which cannot be explained by the Townsend theory either.

\section{STREAMER THEORY}

The theory of streamer discharges dating back to 1939-1940 (Refs. 30-33) quantitatively explains the experimental observations of a self-propagating streamer at high $p d$ in a non-uniform electric field. The streamer theory includes four main aspects, i.e., (1) the first electron for the primary avalanche is due to cosmic radiation or other initial source, (2) for a positive streamer, photoionization in front of the streamer head is the main source of the secondary electrons for the secondary electron avalanche, (3) electron collision ionization is the main ionization mechanism, and (4) the electric field from space charge is comparable to the background electric field. According to the streamer theory, many experimental observations for $p d>200$ Torr $\cdot \mathrm{cm}$ can be well interpreted. The randomness of the generation of the first electron results in the ignition delay and ignition jitter. Moreover, the uncertainty of the photoionization leads to the inconsistency of the propagation speed of a streamer and also to the branching behavior. These features of the streamers have attracted considerable attention in last few decades.

The streamer theory has since become the fundamental base to describe discharges operated under high $p d$ conditions.
Later work focused on the ignition conditions, propagation speed, dimensions, number and radius of branching, the effects of photoionization, and vacuum ultraviolet spectroscopy illumination, addition of radioactive gas, dielectric barrier, effects of the polarity, the rising time, and the pulse width of the applied voltage of the external circuit, as well as the effects of gas temperature and composition, and several other factors. $^{34-77}$

Among all the characteristics of streamers, two of them have attracted particular attention, i.e., (1) the branching behavior and (2) the effect of photoionization, background ionization, and frequency of the applied voltage. In the following, recent advances on the understanding of these two effects will be discussed in more detail.

\section{A. The branching behavior}

The branching behavior has been observed in a large number of experiments. Random appearance of streamers both in time and space suggests that seed electrons may also be randomly generated next to the streamer head. Negative streamers rely on the transport of electrons from the ionized zone into the non-ionized zone in front of the streamer head to provide seed electrons for the avalanche. ${ }^{78-82}$ On the other hand, in the absence of pre-ionization (such as due to the previous discharge pulses or radiation from cosmic rays), positive streamer need photoionization to provide these seed electrons. ${ }^{83-86}$

Recently, substantial efforts have been devoted to control streamer branching. ${ }^{87,88}$ For example, by using a $\mathrm{KrF}$ laser to illuminate a positive streamer, it was found that the positive streamer branching is suppressed in the irradiated region when the intensity of the laser pulse is higher than $1.9 \times 10^{5} \mathrm{~W} \mathrm{~cm}^{-2}$, which corresponds to the ionization density of about $5 \times 10^{5} \mathrm{~cm}^{-3}$. 87

A similar experiment was carried by using $\mathrm{X}$-ray radiation in $\mathrm{F}_{2}$ excimer laser gas mixtures $\left(\mathrm{He}+\mathrm{F}_{2}\right.$ mixtures with various ratios). It was found that at pre-ionization levels of $10^{7} \mathrm{~cm}^{-3} \mathrm{bar}^{-1}$ and higher the discharge was diffuse (uniform) and at lower pre-ionization levels, the discharge became filamentary or streamer-like. ${ }^{88}$

On the other hand, simulation of the branch behavior is a very challenging task. The experimentally observed branching behavior cannot be described numerically if pure fluid models are used. ${ }^{81,82,89,90}$ Until recently, only a few studies on the simulation of the branching behavior of both positive and negative streamers have been reported. 
The branching of positive streamer coaxial discharges in atmospheric pressure air was simulated from the viewpoint of statistical photon transport and photoionization. The statistical photon transport model, which is based on randomly selected emitting angles and mean-free-path for absorption, was embedded into a fluid-based plasma transport model. The results show that secondary streamers, often spatially isolated, are triggered by the random remote photo-electron production which initiates a back-traveling electron avalanche and eventually leads to streamer branching as shown in Fig. 6. In this particular case, there are five primary streamers, labeled from A to E. All streamers propagate nearly in the radial direction. However, they have significantly different propagation
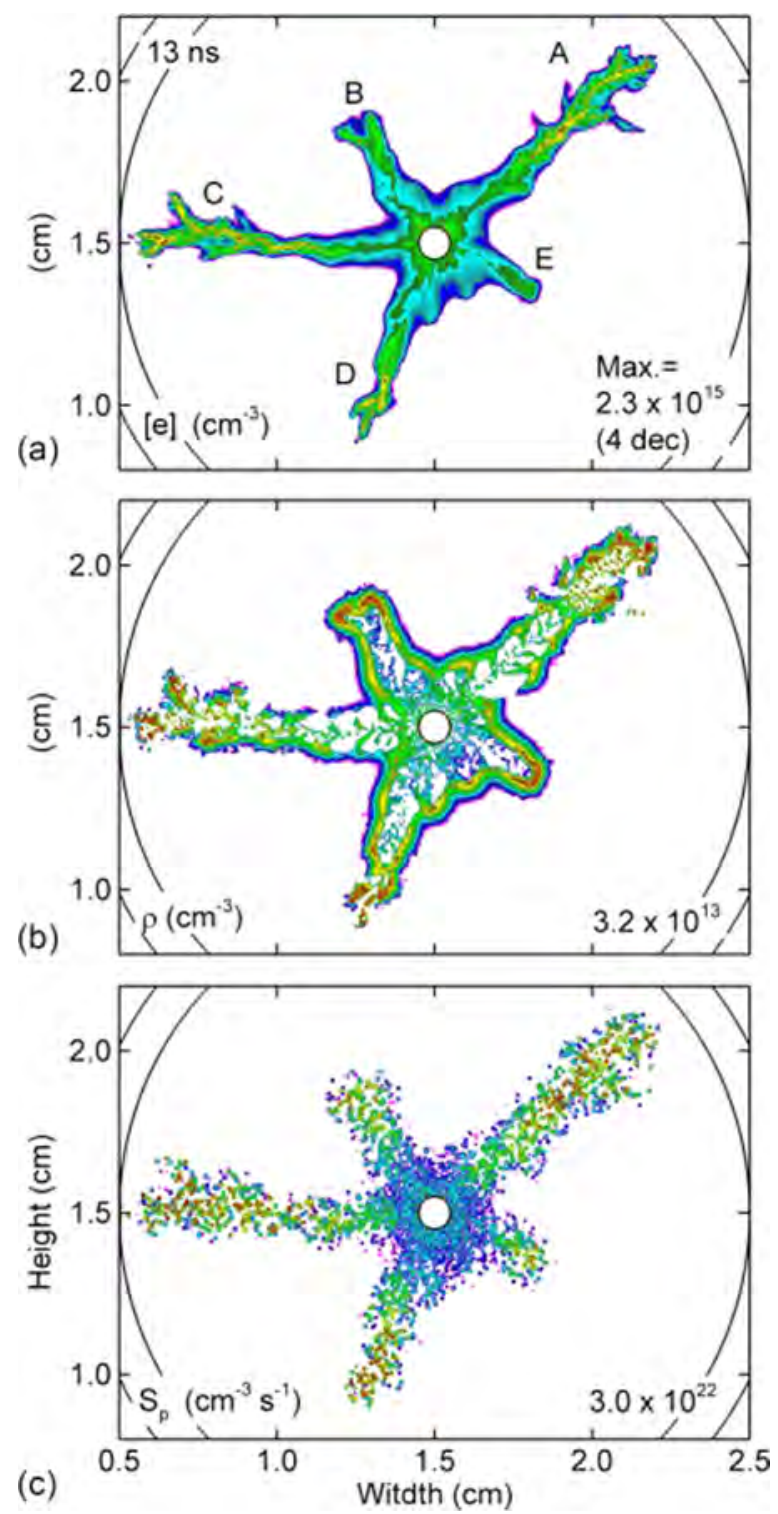

FIG. 6. The propagation, path-deviation, and branching of individual streamers from the statistical photon transport model shown by (a) electron density $n_{\mathrm{e}}$, (b) space charge density $\rho$, and (c) photoionization rate $S_{\mathrm{p}}$ at $t=12.8 \mathrm{~ns}$. Of the five individual streamers, four (A-D) show path-deviation and branching, while streamer E propagates as a single structure. The branching is associated with the breaking of the space charge layer (SCL). Contours are on a log scale over 4 decades. ${ }^{91}$ Reproduced with permission from Z. Xiong and M. J. Kushner, Plasma Sources Sci. Technol. 23, 065041 (2014). Copyright 2014 Institute of Physics. speeds and shapes that differ from the deterministic model predictions. $^{91}$

Besides, a negative streamer in air without photoionization at standard temperature and pressure has recently been studied using particle and hybrid models. The spatially hybrid model follows the particle dynamics only in the dynamically relevant region. Therefore, this model can continue tracking single-electron fluctuations much longer than the particle model. As shown in Fig. 7, the streamers in the particle and in the hybrid model both clearly show branching behavior. However, the branching behavior cannot be reproduced when the fluid model is used. ${ }^{82}$

To ease the computational demands, in most simulations, the photo-ionization in air was replaced by background ionization in several studies. ${ }^{92,93}$ The background electron density $^{81,93-95}$ ranging by the order of magnitude from $10^{2}$ to $10^{10} \mathrm{~cm}^{-3}$ was used in these calculations. Importantly, it was found that the background ionization density has a small influence on negative streamers but has a large influence on positive streamers. ${ }^{81,92-95}$

\section{B. Effect of photoionization}

As mentioned above, one of the fundamental aspects of the positive streamer theory is that photoionization in front of the streamer head is the main source of the secondary electrons for the secondary electron avalanche. This is the case for single shot discharges or discharges with very low frequency. If the repetition frequency is high enough, the residual electrons left from the previous discharge or due to the detachment of $\mathrm{O}_{2}{ }^{-}$when a significant amount of $\mathrm{O}_{2}$ molecules is present in the working gas could play the same role as electrons produced by photoionization and thus affect the propagation of the streamer. However, the minimum concentration of residual electrons to dominate over photoionization effects in the streamer propagation is still unknown. To resolve this issue, numerical modeling with or without inclusion of photoionization, and with different levels of background ionization has been carried out. It is worth pointing out that the simulations mainly focus on the effects of photoionization and background electron density on the streamer structure and propagation speed. We are not aware of the studies of the effect of photoionization and background electron density on the streamer ignition delay and jitter.

Because of the many intrinsic difficulties, there is only a limited number of experimental works on direct measurements of photoionization; many of these studies were carried several decades ago. ${ }^{96-102}$ For example, one study found out that the photoionization in air is about 1 to 2 orders of magnitude more effective than in pure oxygen or pure nitrogen, but they still see significant photoionization in both pure gases. However, the purities of the gas are probably on the order of $0.1 \%-1 \%$. In other words, air is still present in the "pure" gas. ${ }^{102,103}$

On the other hand, many numerical studies focusing on the role of photoionization in the propagation of traditional streamers have been reported. These studies investigated the effect of the applied electric field, gas pressure, and composition on photoionization, as well as the effect of photoionization 

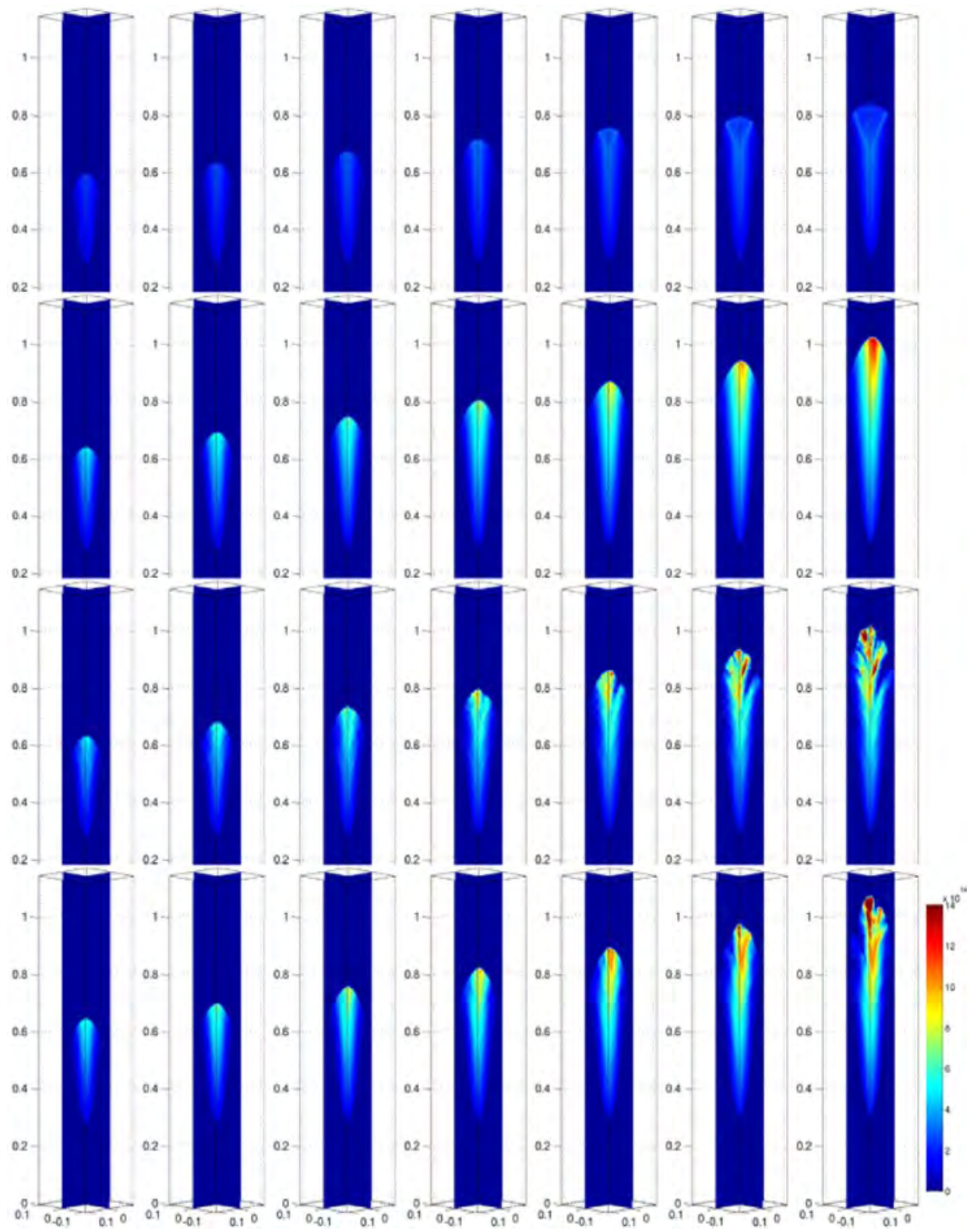

FIG. 7. The electron density distribution obtained from the extended fluid model (first row), particle model (second row), and hybrid model (third row). ${ }^{82}$ Reproduced with permission from Li et al., Plasma Sources Sci. Technol. 21, 055019 (2012). Copyright 2012 Institute of Physics.

on streamer characteristics, including the branching, thickness, number/density, and propagation velocity of the streamers. ${ }^{83,84,92,104-113}$ Besides, background ionization was suggested as an alternative mechanism to photoionization. This suggestion was even used to reduce the computational complexity by replacing photoionization by the equivalent level of background ionization. ${ }^{92}$

In order to quantify the effect of photoionization on streamer propagation, photoionization model is needed. One of the well-known and widely used photoionization models ${ }^{114}$ was developed in 1982 based on the experimental results reported decades ago. ${ }^{96-101}$ The rate of photoionization in this model is represented as

$$
S_{p h}(r)=\frac{\xi}{4 \pi} \frac{p_{q}}{p+p_{q}} \int \frac{h\left(p\left\lfloor r-r^{\prime}\right\rfloor\right) S_{i}\left(r^{\prime}\right) d^{3}\left(p r^{\prime}\right)}{\left\lfloor p r-p r^{\prime}\right\rfloor^{2}}
$$

where $\xi$ is a proportionality constant, $p$ is the gas pressure, $p_{q}=80$ mbar is the quenching pressure, $S_{i}$ is the local impact ionization rate of nitrogen, and $h$ is the absorption function of the ionizing photons. Because integral expressions are computationally costly, a two-term approximation was introduced $^{115}$

$$
S_{p h}=\frac{p_{q}}{p+p_{q}} \sum_{j=1}^{N} A_{j} S_{p h, j}, \quad\left(\nabla^{2}-\lambda_{j}^{2}\right) S_{p h, j}=S_{i},
$$

where coefficients $A_{j}$ and $\lambda_{j}$ are chosen to fit the experimental model, and $\lambda_{j}$ is related to the characteristic absorption length, while $A_{j}$ represents the intensity. When $\mathrm{N}=2$, this model is named as the Luque two-term model, where $\mathrm{A}_{1}=4.6 \times 10^{-2} \mathrm{~cm}^{-1}$ bar $^{-1}, \quad \mathrm{~A}_{2}=2.7 \times 10^{-3} \mathrm{~cm}^{-1} \mathrm{bar}^{-1}$, $\lambda_{1}=45 \mathrm{~cm}^{-3}$ bar $^{-1}$, and $\lambda_{2}=7.6 \mathrm{~cm}^{-3} \mathrm{bar}^{-1}$. Bourdon et al. ${ }^{116}$ suggested that the 2-term model for photoionization is insufficient and proposed to replace it by a similar model with 3 terms; the latter model was named as the Bourdon's three-term model.

However, these models are based on early experimental works. Because of the uncertainty of the experimental conditions, the actual parameters of photo-ionization are not 
known. In order to determine the sensitivity of the simulation results on the parameters used in the photo-ionization models, simulations of streamers in air without background ionization in three cases were carried out. ${ }^{117}$ The first case is based on the Luque's two-term approximation model. The second case is based on the Bourdon's three-term model. The final test-case uses the default 2-term model, but with the number of emitted photons artificially reduced by a factor of 10 . The positions of the streamer heads as a function of time are plotted in Fig. 8. One can see that the difference between the Luque-model and the artificially weakened Luque-model is not very large. Indeed, the "weakened photoionization" scenario has 10 times less source electrons in front of the streamer head, but it only takes $20 \%$ longer time to cross the gap between the electrodes. The results with Bourdon's 3-term model lie between the other two curves.

It is worth pointing out that for the widely accepted photoionization model discussed above, it is assumed that the major contribution to the rate of photoionization is nitrogen radiation in the spectral range of $98-102.5 \mathrm{~nm}$. Here, $102.5 \mathrm{~nm}$ is the photoionization threshold of $\mathrm{O}_{2}{ }^{58}$ Below $98 \mathrm{~nm}$, the radiation is strongly absorbed by nitrogen and gives a minor contribution to photoelectron production. ${ }^{118}$ One could thus expect that the photoionization rates should strongly depend on the $\mathrm{O}_{2}$ percentage. However, experimental results found out that several important streamer properties such as streamer diameters and velocities are quite similar for nitrogen-oxygen mixtures with nearly six orders of magnitude difference in oxygen fraction and pure nitrogen with an impurity level of less than $1 \mathrm{ppm}$. The streamers propagate with roughly the same velocity and their minimal diameter decreases by less than a factor of 2 from artificial air to pure nitrogen.

This means that if photoionization is important in the streamer propagation, either (i) other photo-ionization mechanisms than direct photo-ionization of oxygen by nitrogen

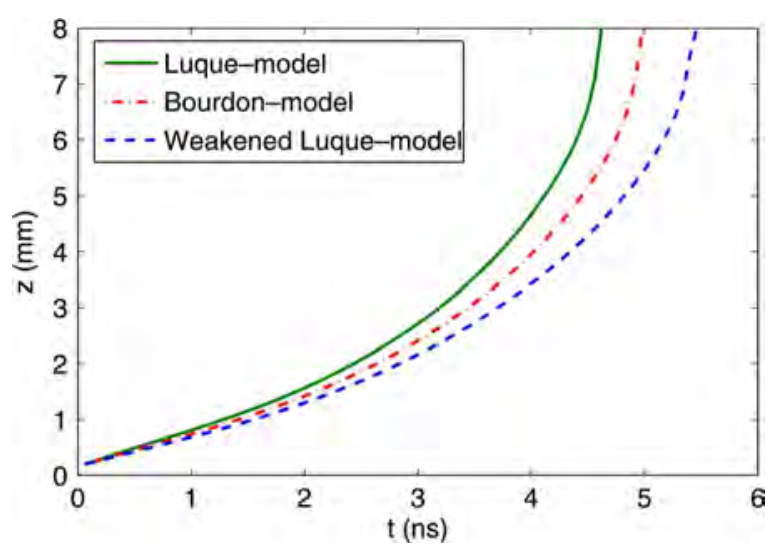

FIG. 8. Comparison of three photo-ionization models describing the position of the streamer head. The curve labeled "Luque model" corresponds to Luque two-term model. The curve labeled "Bourdon-model" corresponds to Bourdon 3-term model. The bottom curve uses the Luque-model, where the source term for the photoionization equation was artificially reduced by a factor of 10. Such "weakened photoionization" scenario does not represent an actual physical scenario; its purpose is to demonstrate the influence of the accuracy of the photo-ionization parameters. ${ }^{117}$ Reproduced with permission from Wormeester et al., J. Phys. D: Appl. Phys. 43, 505201 (2010). Copyright 2010 Institute of Physics. emission are responsible for free electrons generation (such as step-wise ionization of nitrogen molecules) or (ii) background ionization of the gas due to cosmic radiation, radioactivity or leftover charges from previous discharges can deliver enough free electrons for streamer propagation. ${ }^{113,117}$ However, the frequency used in this experiment is between $0.03 \mathrm{~Hz}$ and $1 \mathrm{~Hz}$. Thus, the leftover charges from previous discharges must be too low to play an important role in the streamer propagation. The background ionization can lead to free or bound electrons. The bound electrons can be detached by the enhanced electric field of the streamer head. ${ }^{113}$

In order to elucidate the effect of photoionization on the streamer propagation, it is important to estimate the electron density due to photoionization. Simulation results obtained with various initial seed ionizations showed that without photoionization, an initial seed electron density of $10^{7} \mathrm{~cm}^{-3}$ can lead to similar streamer parameters as in the case when photoionization is included in the model without the initial seed ionization. Consequently, the electron density from the photoionization is on the order of $10^{7} \mathrm{~cm}^{-3} .119$

Interestingly, photoionization is responsible for the rather unexpected streamer attraction phenomenon. ${ }^{120}$ Besides, simulations showed that the photoionization process is one of the most critical factors contributing to non-similar behavior of streamers propagating in strong homogeneous external electric fields. The intensity of the field should satisfy $E>E_{k}$, where $E_{k}$ is the conventional breakdown threshold field defined by the equality of the ionization and dissociative attachment coefficients in air. ${ }^{112}$ Moreover, it was found that quenching of singlet excited states of molecular nitrogen emitting photo-ionizing radiation is responsible for the nonsimilar behavior of short streamers in air. ${ }^{108,112,121} \mathrm{We}$ emphasize that the modelling results and their interpretation based on non-similarity of the photoionization process at high pressures in air are consistent with the experimental observations, where streamers have more and thinner channels and branch more frequently at higher (i.e., near atmospheric) pressures than at lower pressures.

\section{Effect of seed electron density}

For a positive streamer, seed electrons are needed in order to propagate. The other source of seed electrons is the residual electrons left from the previous discharge or due to detachment of $\mathrm{O}_{2}{ }^{-}$under high electric field. Obviously, the residual electrons only exist when discharges occur under certain frequency. For a given discharge, it might be possible to obtain the electron density immediately after the discharge. Then by using kinetic simulation of electrons, the residual electron density can be estimated for a given frequency. It is usually expected that when the frequency is high enough, the discharges will transit from a stochastic mode to a repeatable mode. However, because of the limited availability of high voltage power supplies with high frequency, for all the experimental works that have been reported, the highest frequency was only $10 \mathrm{~Hz}$. The latter frequency is too low for a discharge to appear repeatable in atmospheric pressure air. 
In order to better understand the role of seed electron density on the propagation of streamers, computer simulations are a powerful tool and have been widely used. To simplify the computations, the background electron density is commonly directly input in the simulations, and its value varies in a wide range from $\sim 10^{2}$ to $\sim 10^{10} \mathrm{~cm}^{-3} \cdot{ }^{81,92-95}$

Figure 9 presents the results of modeling of positive and negative streamers propagating between two-point electrodes with different seed charges in atmospheric pressure air. ${ }^{94}$ It shows the axial profiles of the electron density at $t=2 \mathrm{~ns}$ for 6 values of the pre-ionization background. One can note that the significant decrease in the pre-ionization background from $\sim 10^{9}$ to $\sim 10^{4} \mathrm{~cm}^{-3}$ has only a small influence on the electron density of the negative streamer head. Conversely, the decrease in the pre-ionization background shows a significant influence on the positive streamer characteristics. For a pre-ionization background of $\sim 10^{9} \mathrm{~cm}^{-3}$, the positive streamer starts propagating immediately. But when the preionization level decreases, the delay for the positive streamer starts increasing.

Besides, the results obtained for a pre-ionization level of $10^{5} \mathrm{~cm}^{-3}$ without photoionization are also presented in Fig. $9 .{ }^{94}$ For a seed electron density (SED) higher than $10^{7} \mathrm{~cm}^{-3}$, both positive and negative streamers propagate with the SED level ahead of both streamer fronts remaining at the same level as at the point of discharge initiation $t=0$. However, for the seed electron density less than $10^{7} \mathrm{~cm}^{-3}$, it was found out that seed electrons created by photoionization ahead of the negative streamer front contribute to the increase in seed charges in the inter-electrode gap, thereby sustaining the propagation of the positive streamer head. These results thus indicate that the electron density induced by photoionization is on the order of $10^{7} \mathrm{~cm}^{-3}$.

Figure 10 shows the results of numerical modeling of positive and negative streamers in a needle-plane geometry for different seed electron density. ${ }^{95}$ The seed electrons are

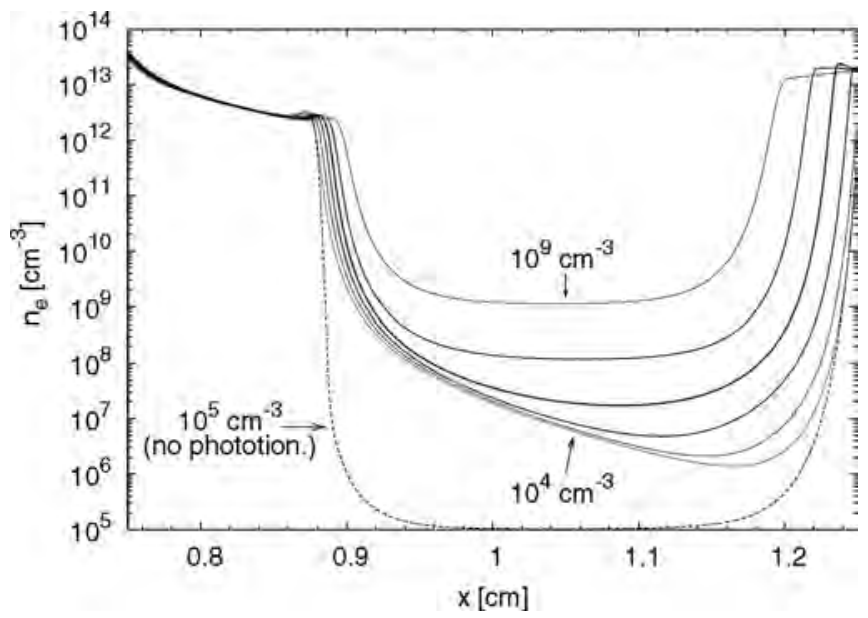

FIG. 9. Electron density between two-point electrodes along the axis of symmetry at $t=2 \mathrm{~ns}$ for various pre-ionization backgrounds at $t=0$. The left side is the cathode, while the right side is the anode. Solid lines: Results are shown for pre-ionization background values from $10^{4}$ to $10^{9} \mathrm{~cm}^{-3}$, with a multiplier step of 10. Dashed line: Results obtained for a pre-ionization background of $10^{5} \mathrm{~cm}^{-3}$, without photoionization. ${ }^{94}$ Reproduced with permission from Bourdon et al., Plasma Sources Sci. Technol. 19, 034012 (2010). Copyright 2010 Institute of Physics.

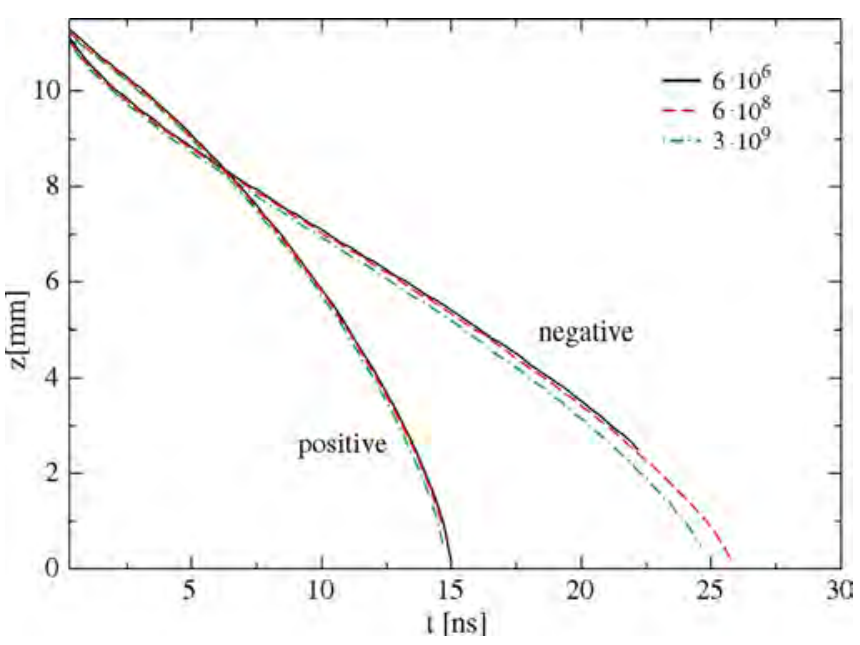

FIG. 10. Front propagation of negative and positive streamers for different seed electron density between needle-plane electrodes. The gap is $L=11.5 \mathrm{~mm}$ long with an applied voltage of $23 \mathrm{kV}$; the needle parameters are $L_{\text {needle }}=2.3 \mathrm{~mm}$ and $R_{\text {needle }}=0.26 \mathrm{~mm}$. Different line styles correspond to different SED values. ${ }^{95}$ Reproduced with permission from Luque et al., J. Phys. D: Appl. Phys. 41, 234005 (2008). Copyright 2008 Institute of Physics.

placed at the top of the gap next to the needle and have spherical Gaussian profiles with a radius of $92 \mu \mathrm{m}$ and the number of electrons between $6 \times 10^{6}$ and $3 \times 10^{9} \mathrm{~cm}^{-3}$. One can see that the streamer propagation only weakly depends on the initial conditions. It should be emphasized that the initial seed electrons are only placed at the region next to the needle electrode, while the rest of the space has no seed electrons. The reason why the propagation of the streamers only weakly depends on the initial seed electrons might be due to the rapid growth of SED in the high field region near the needle electrode.

Since $\mathrm{O}_{2}{ }^{-}$could contribute significantly to the seed electrons, simulations for different initial $\mathrm{O}_{2}^{-}$density without including photoionization effects have been conducted. These results are also compared with the case when photoionization is included without any initial background ionization. Figure 11 shows the competition of photoionization and

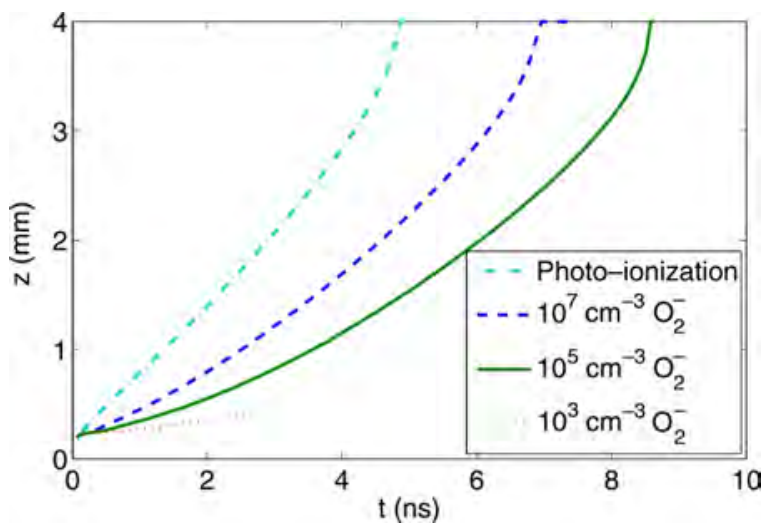

FIG. 11. Position of the streamer head as a function of time in the needle-toplane geometry. The top curve corresponds to an air streamer with photoionization (scenario 1), the curves below that to streamers without photoionization, and with a decreasing amount of uniform background $\mathrm{O}_{2}{ }^{-}$concentrations (scenarios 2, 3, and 4). The $z=0$ point corresponds to the tip of the needle. ${ }^{117}$ Reproduced with permission from Wormeester et al., J. Phys. D: Appl. Phys. 43, 505201 (2010). Copyright 2010 Institute of Physics. 
background $\mathrm{O}_{2}{ }^{-}$concentration. ${ }^{117}$ Without photoionization, the streamer is able to propagate only if a sufficiently high level of background $\mathrm{O}_{2}{ }^{-}$exists in front of the streamer head. The streamer propagates about $40 \%$ faster with photoionization than with a background $\mathrm{O}_{2}{ }^{-}$concentration of $10^{7} \mathrm{~cm}^{-3}$, which roughly corresponds to a discharge with a repetition frequency of $1 \mathrm{~Hz}$ in atmospheric pressure air. When the background $\mathrm{O}_{2}{ }^{-}$concentration decreases by a factor of 100 from $10^{7}$ to $10^{5} \mathrm{~cm}^{-3}$ (corresponding to a frequency of $0.01 \mathrm{~Hz}$ ), the streamer's propagation speed decreases by only $20 \%$. However, a background $\mathrm{O}_{2}{ }^{-}$concentration of $10^{3} \mathrm{~cm}^{-3}$ was not sufficient to start a streamer that would propagate more than a few hundred micrometers. To find the minimum $\mathrm{O}_{2}{ }^{-}$concentration to play a similar role as the photoionization, the initial $\mathrm{O}_{2}{ }^{-}$concentrations were further increased.

As shown in Fig. 12, while the $\mathrm{O}_{2}{ }^{-}$density is at a level of $10^{11} \mathrm{~cm}^{-3}$, the negative oxygen ions start increasing the streamer velocity. ${ }^{117}$ Since $\mathrm{O}_{2}{ }^{-}$concentration varies from 0 to $10^{9} \mathrm{~cm}^{-3}$, the streamers propagate due to photoionization and are insensitive to the additional background concentration of $\mathrm{O}_{2}^{-}$. In other words, the photoionization mechanism dominates the streamer propagation except when the background $\mathrm{O}_{2}{ }^{-}$concentration is higher than $10^{9} \mathrm{~cm}^{-3}$. If we assume that all the $\mathrm{O}_{2}{ }^{-}$are neutralized and the detached electrons join the seed electrons, then the electron density due to photoionization should be on the order of $10^{9} \mathrm{~cm}^{-3}$. In reality, not all the electrons will detach from $\mathrm{O}_{2}{ }^{-}$. We thus conclude that the electron density due to photoionization should be less than $10^{9} \mathrm{~cm}^{-3}$.

\section{Effect of frequency of the applied voltage}

From the experimental point of view, the effects of background ionization/leftover ionization from the prior discharges on a new discharge can be investigated by changing the frequency of the applied voltage pulses. This is because, with the change of discharge frequency, the pulse off time is changed. This change in turn affects the background electron density due to attachment, recombination, diffusion, charge transfer, and some other processes. However, it is experimentally extremely challenging to build a pulsed power supply

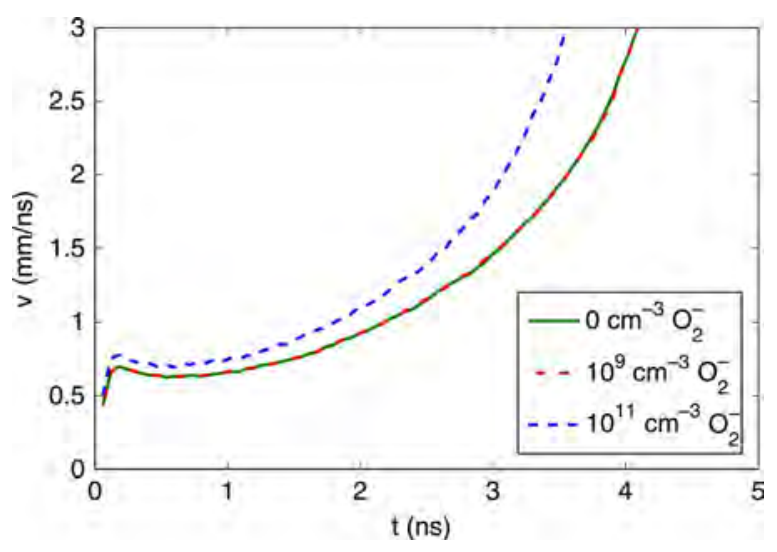

FIG. 12. Propagation speed of streamers in air with different levels of background $\mathrm{O}_{2}{ }^{-}$concentration. Photo-ionization is present in all three cases. ${ }^{117}$ Reproduced with permission from Wormeester et al., J. Phys. D: Appl. Phys. 43, 505201 (2010). Copyright 2010 Institute of Physics. with a wide range variable repetition frequency while maintaining the same pulse shape and amplitude. This is why most of the works reported are based on the results of individual discharges or a single-shot discharge. Only a few works have been reported on the effect of background electron density on characteristics of the streamers by adjusting the discharge frequency. As mentioned above, the maximum frequency was only $10 \mathrm{~Hz}$. For example, at frequency of $5 \mathrm{~Hz}$, in a $100 \mathrm{mbar}$ discharge in pure $\mathrm{N}_{2}$, it was found that the build-up of background ionization affects the morphology of subsequent discharges although the streamers of the subsequent discharges do not follow exactly the same path of the previous discharges. ${ }^{122}$

Since it is experimentally extremely challenging to build a pulse source with a wide range variable repetition frequency while maintaining the same pulse shape and amplitude, on the other hand, to build a pulsed power supply having only two pulses with the variable time interval in a wide range is much less challenging. Besides, as we know that the life time of electrons and ions at atmospheric pressure air is very short, thus the difference of the densities of residual electrons and ions between a single preceding pulse and a sequence of preceding pulses might not be large. However, if long lifetime reactive species, such as metastable state species, also contribute to the generation of electrons and ions, then they might cause a large difference. Based on such point of view, recently, by applying two subsequent pulses with pulse-to-pulse intervals $\Delta t$ between $200 \mathrm{~ns}$ and $40 \mathrm{~ms}$, the effect of the first discharge on the second discharge was investigated. ${ }^{50}$

An example of the photos of the two discharges in 133 mbar air with a large variation $\Delta t$ is given in Fig. $13 .{ }^{50}$ The discharges generated by the first pulse are, except for stochastic variations, similar for all values of $\Delta t$. The development of the second discharge pulse as a function of $\Delta t$ can be divided into several stages. The streamers ignited by the second pulse start propagating from the tip of the primary streamer when the pulse-to-pulse intervals are $600 \mathrm{~ns}$ or less. This indicates that the primary streamer has a relatively high conductivity at the point when the second pulse starts. When the pulse-to pulse intervals are further increased up to $1.8 \mu \mathrm{s}$, the streamers ignite from the tip electrode. New streamer channels appear while avoiding the old streamer pathways when the pulse-to-pulse intervals are increased further to 2.8 $\mu \mathrm{s}$. When the pulse-to-pulse intervals increase to $3.3 \mu \mathrm{s}$, more new channels appear. Some of the new channels follow the edges of the old channels. They start to overlap more with the channels generated during the first pulse when the pulse-to-pulse interval increases to $6.8 \mu \mathrm{s}$ and a large number of the second-pulse streamers still follow the first-pulse streamer paths even when the pulse-to-pulse interval is increased to $1 \mathrm{~ms}$. Only when the pulse-to-pulse interval is increased to around $10 \mathrm{~ms}$, the second discharge appears fully independent of the first discharge. In other words, only when $\Delta t$ is around $10 \mathrm{~ms}$, the residual effect of the first pulse completely disappears. In this case, the concentrations of the electrons, residual ions, excited metastable, and other species, are too low to have any effect on the second discharge pulse. 

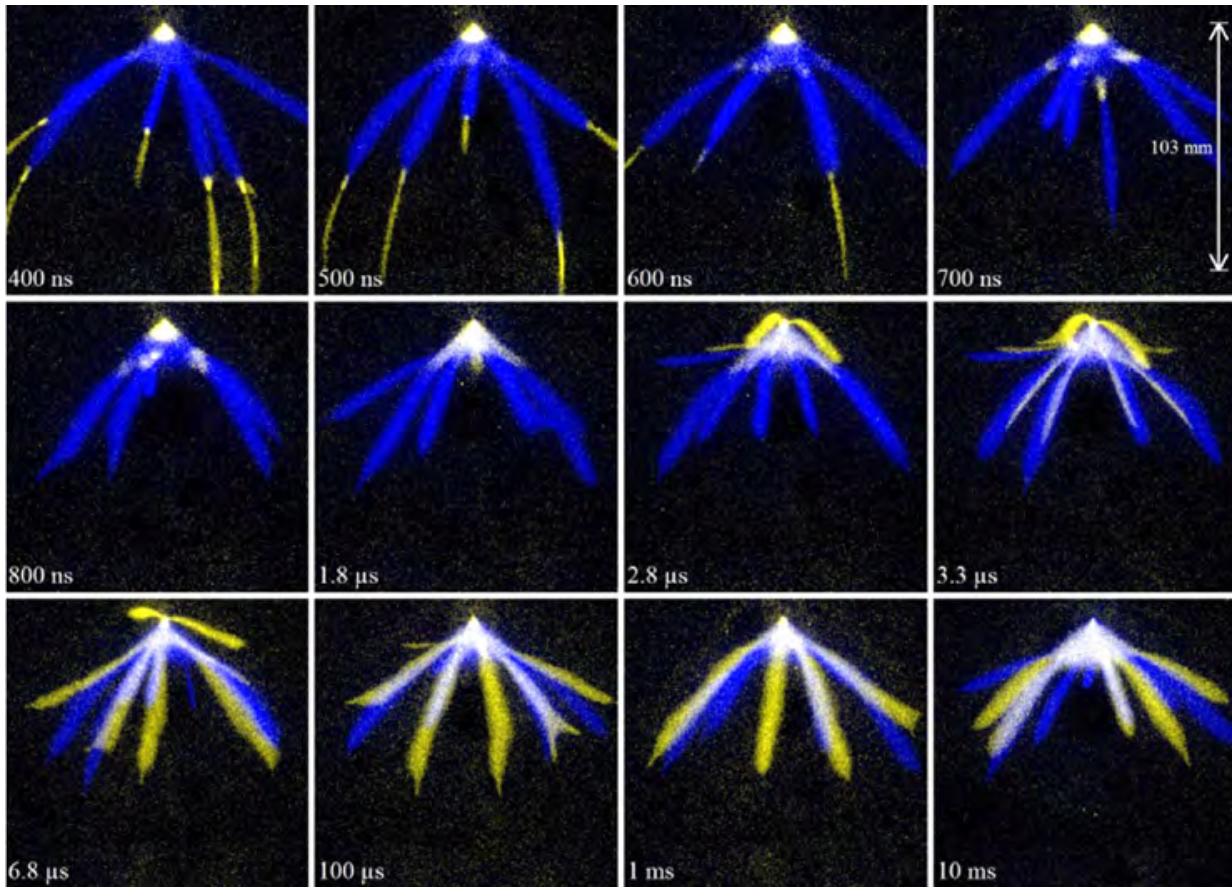

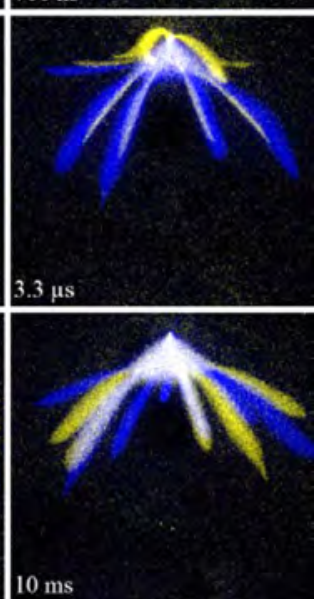

FIG. 13. Superimposed discharge-pair images for varying pulse-to-pulse interval $\Delta t$ as indicated in the images. Images taken in ${ }^{133}$ mbar artificial air with pulses of $13.6 \mathrm{kV}$ amplitude and $200 \mathrm{~ns}$ pulse length. Areas that only emit during the first pulse are blue, areas that only emit during the second pulse are yellow, and areas that emit during both pulses are white. $^{50}$ Reproduced with permission from Nijdam et al., Plasma Sources Sci. Technol. 23, 025008 (2014). Copyright 2014 Institute of Physics.
There are few points that should be discussed in this regard. First, the above study only investigated the effect of the first pulse on the second pulse. ${ }^{50}$ When the streamers are operated in a continuous mode, the integrated effect from precedent multiple pulses could be very different from the effect of the single pulse. Second, the gas pressure of the discussed study was 133 mbar. Consequently, at atmospheric pressure, the pulse-to-pulse intervals corresponding to the different stages could also be different.

\section{E. Effect of gas pressure}

Besides the discharge frequency, the gas pressure and the amplitude of the applied voltage could also affect the background ionization and thus the characteristics of the streamers. Figure 14(a) shows the streamer stagnation time, which is the time interval between the discharge inception and the breakup into streamers, as a function of pressure in air for different voltage amplitudes. ${ }^{123}$ The streamer stagnation time roughly scales as $\sim 1 / p$ regardless of the voltage. Jitter of the stagnation time decreases with voltage and increases with gas pressure. Figure 14(b) shows that the streamer stagnation time is almost the same for the three voltage amplitudes for the same discharge frequency of $1 \mathrm{~Hz}$ and the jitter does not decrease much with the voltage. ${ }^{123}$ The influence of the repetition frequency is also shown in Fig. 14(b). The average stagnation time is quite similar for the three repetition rates. But the jitter of the stagnation time decreases with the repetition frequency. ${ }^{123}$ It is worth emphasizing that the jitter of the stagnation time for all the cases reported here is tens of nanoseconds or even longer; they are much longer than in the case of plasma bullets when they propagate in a repeatable mode, as will be discussed in Sec. IV.

\section{F. Effect of adding radiative gas}

In addition, the background ionization can also be controlled by adding radiative gas. Studies estimated that adding $9.9 \mathrm{ppb}$ of ${ }^{85} \mathrm{Kr}$ to pure nitrogen at 200 mbar could create a background ionization level of about $4 \times 10^{5} \mathrm{~cm}^{-3} \cdot{ }^{21}$ It was found that it starts affecting the streamer morphology in the ${ }^{85} \mathrm{Kr}$ mixture only at repetition rates of $1 \mathrm{~Hz}$ or higher. Therefore, it was concluded that the background ionization level should be close to $4 \times 10^{5} \mathrm{~cm}^{-3}$ for a discharge frequency of $1 \mathrm{~Hz}$ in pure $\mathrm{N}_{2}$ at 200 mbar. This interesting result confirmed that background ionization levels indeed play an important role in streamer propagation.

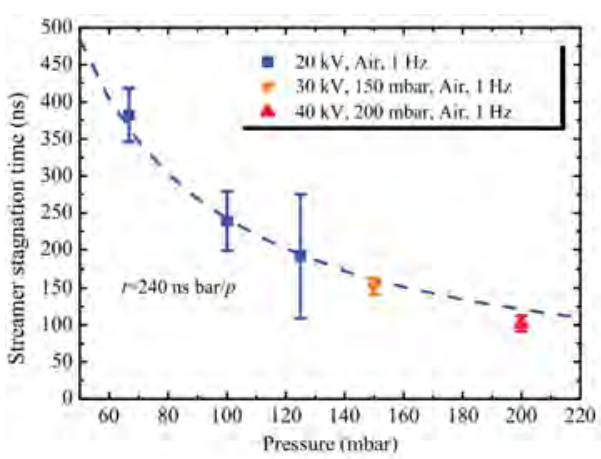

(a)

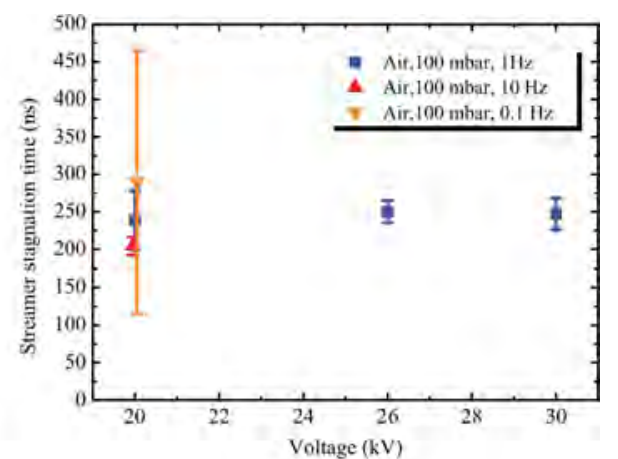

(b)
FIG. 14. (a) Average streamer stagnation time with standard deviation in air as a function of pressure for different voltage amplitudes. (b) Average stagnation time with standard deviation in air as a function of voltage for different pulse repetition frequencies. ${ }^{123}$ Reproduced with permission from Chen et al., J. Phys. D: Appl. Phys. 48, 175201 (2015). Copyright 2015 Institute of Physics. 


\section{G. Preliminary summary}

In short, electron density induced by photoionization should be on the order of $10^{7}-10^{8} \mathrm{~cm}^{-3}$ for a streamer to propagate. Simulations show that only when the concentration of $\mathrm{O}_{2}^{-}$is higher than $10^{9} \mathrm{~cm}^{-3}$, which corresponds to a discharge frequency of $1 \mathrm{kHz}$ in atmospheric pressure air, the background ionization starts to play a significant role. On the contrary, photoionization plays the dominant role in the propagation of positive streamers. On the other hand, most of the experimental results reported are based on single-shot studies. Few of these studies use repetitive discharges, and the maximum frequency is up to $10 \mathrm{~Hz}$. Besides, the gas pressure is a few hundred mbar rather than atmospheric pressure. This is due to the extraordinary challenge of building a suitable high voltage power supply. In 100 mbar pure $\mathrm{N}_{2}$, it was found that the build-up of background ionization has influenced the morphology of the streamers at a frequency of several Hz. In 100 mbar air, the jitter of the streamer stagnation time decreases when the repetition frequency is increased from 0.1 to $10 \mathrm{~Hz}$. Studies from two-pulses-system in 133 mbar air found that the first pulse still affects the second pulse when the pulse-to-pulse interval is less than $10 \mathrm{~ms}$. In other words, when the discharge frequency is lower than $100 \mathrm{~Hz}$, the concentrations of the electrons, residual ions, and excited species in metastable states are too low to have any effect on the second discharge pulse.

Because of the nature of streamers, fast propagation, and poor repeatability from pulse to pulse, it is extremely difficult to understand the physics behind some of the phenomena. From the experimental point of view, due to the limited availability of suitable pulsed power supplies and also due to the lack of reliable diagnostic systems, the understanding of the streamers in air is still limited. It is not known if it is possible, or if possible, when and how the streamer behavior changes from non-repeatable to repeatable. On the other hand, the plasma bullet behavior of atmospheric pressure plasma jets, which is a kind of streamer discharges, features high repeatability, i.e., propagates the same distance after the same delay time. Such an exotic characteristic is very useful to understand the physics of streamers, in general. In addition, because the working gas of the plasma jets is a noble gas, such as $\mathrm{He}$ and Ar, which has a much lower breakdown voltage, it is much less challenging to build a pulsed power supply with a wide range of frequency at a relatively low voltage. In the following, the recent studies of the effect of photoionization and seed electron density on the repeatability of plasma bullets of atmospheric pressure plasma jets are critically examined.

\section{GUIDED IONIZATION WAVE: HIGH SEED ELECTRON DENSITY (HSED) DISCHARGE}

As discussed in the Introduction, plasma bullets behave differently from traditional streamer discharges. The streamer theory cannot interpret many experimental observations related to plasma bullets. One of the significant differences is the reproducibility of the plasma bullets which propagate the same distance after the same delay time. $9,19,40,43,124-170$ On the other hand, it is noticed that most N-APPJs are driven in noble working gases by $\mathrm{kHz}$ or higher frequency power supply. This is different from the streamers, which are mainly single shot or several hertz only with air as working gas. As we know, the electron density decrease takes place over specific decay times. In air at standard temperature and pressure, the free electrons are captured by $\mathrm{O}_{2}$ molecules during only about ten nanoseconds, forming negative ions. The captured electrons are less effective to contribute to the ionization process unless they are detached under a high electric field. On the other hand, as $\mathrm{N}$-APPJs are mainly operated in noble gases, the lifetime of the free electrons could be much longer. Thus, the seed electron density left from previous discharge pulses could be significantly higher than in traditional streamer discharges.

According to the four aspects of the streamer theory presented above, if the seed electron density in the discharge channel is high, the seed electrons could supply free electrons. These electrons could then be used either as the first electron for the primary avalanche or as the secondary electrons for the secondary electron avalanche. Then, the randomness of the generation of the first electron, which results in the ignition delay and ignition jitter, and the uncertainty of the photoionization, which leads to the inconsistency of the propagation speed of a streamer and also to the branching behavior, will not exist anymore. If appropriate conditions are met, the dynamics of the plasma plume will be in a repeatable mode, which was observed by many groups.

Now several questions arise:

(1) First, do all plasma bullets have a high seed electron density? In other words, do all plasma bullets propagate in a repeatable mode? If the answer is no, then when a plasma bullet propagates in an unrepeatable (random) mode, it needs to be answered whether the stochastic mode is due to the inconsistence of the ignition (the first electron for the primary avalanche) of the plasma or the propagation speed (the secondary electrons for the secondary electron avalanche).

(2) Second, for most studies reported, the propagation behavior is captured when the plasma has been running for seconds to minutes or even longer. In these cases, there is accumulation of charged particles and reactive species left from the many previous pulses. On the other hand, for the very first discharge (ignition phase), there are no residual electrons due to the previous discharge, while the seed electron density due to natural radioactivity is very low $\left(\sim 10^{4} \mathrm{~cm}^{-3}\right)$. Thus, the propagation of the plasma bullet for the very first discharge pulses is likely to be different from the stable discharge phase. It will thus be important to confirm if the propagation of the plasma bullet of the very first discharge is not in a repeatable mode. Furthermore, it will be helpful to know after how many discharge pulses the discharge will transfer from a stochastic to a repeatable mode.

(3) Third, what is the minimum seed electron density required for a N-APPJ to propagate in a repeatable mode?

(4) Fourth, what is the electron density due to photoionization and does photoionization play any role in the propagation of N-APPJ? 
To answer questions (1)-(4) above, precise measurements of the seed electron density and the electron density due to photoionization would be particularly useful. However, since the electron density $n_{e}$ of the plasma is numerically estimated to be between $10^{11}$ and $10^{13} \mathrm{~cm}^{-3}$, the seed electron concentration $n_{\text {seed }}$ must be many orders lower than the electron density of the plasma. The seed electron density $n_{\text {seed }}$ assumed by different groups is vastly different, ranging from $10^{2}$ to $10^{10} \mathrm{~cm}^{-3}$. ${ }^{12}$ For such a low electron density, currently there is no reliable experimental method available that can be used to directly measure it. On the other hand, as the seed electron density $n_{\text {seed }}$ decays with time, when the discharge frequency varies, the corresponding time interval between two consecutive discharges changes accordingly. Thus, by reducing the discharge frequency, the discharge must change from a repeatable mode to a stochastic mode when the frequency is lower than the minimum required frequency because the seed electron density is lower than a certain threshold. Based on such assumption, several experiments were conducted to answer the four questions listed above.

\section{A. Plasma bullet propagation: Repeatable and stochastic modes}

First, it was found that not all plasma bullets propagate in a repeatable mode. When the pulse frequency is reduced from 10 to $0.25 \mathrm{kHz}$, the dynamics of the plasma plume do not show any obvious difference. Indeed, the location of the plasma bullets is always the same for the same delay time; this is the key indicator of the streamer propagation in a repeatable mode. However, when the frequency is further reduced to $0.2 \mathrm{kHz}$, the plasma plume still looks the same to the eyes, but the photographs captured by the ICCD camera show that there is a significant difference from the case of $0.25 \mathrm{kHz}$ as shown in Fig. 15. Although the plasma still appears like a bullet for the frequency of $0.2 \mathrm{kHz}$, the location of the plasma bullet is not the same anymore for the same delay time. ${ }^{171}$ The plasma bullets thus propagate in a stochastic mode under such conditions.

Next, when a plasma bullet propagates in the stochastic mode, in order to know whether the stochastic mode is due to the inconsistence of the ignition of the plasma or the propagation speed, two photomultiplier tubes (PMTs) are used to capture the light emission of the plasma plume. ${ }^{172}$ The PMT 1 is placed close to the high voltage electrode and the distance between the PMT 1 and the PMT 2 is $2.5 \mathrm{~cm}$. The time between the peak of the PMT 1 and the rising edge of the applied voltage (50\% of its maximum) is referred as $t_{\mathrm{d}}$, and the time between the PMTs is referred as $t_{\mathrm{v}}$. Since the distance $L$ between the two PMTs is fixed, the propagation velocity of the plasma bullet can be calculated by $\mathrm{v}=L / t_{\mathrm{v}}$. If $t_{\mathrm{v}}$ is the same for the same discharge conditions, it is reasonable to assume that the propagation velocity of the plasma bullet from the electrode to the position of PMT 1 is also a constant. Under such conditions, the ignition delay time $t_{\mathrm{d}}$ can be used to determine the mode of the discharge, i.e., repeatable or unrepeatable/stochastic mode. When the jitter of $t_{\mathrm{d}}$ is less than a certain value, the plasma bullet is considered as propagating in a repeatable mode. Otherwise, it is considered as propagating under an unrepeatable/stochastic mode.

Since a typical propagation velocity of the plasma bullet is on the order of $10^{5} \mathrm{~m} / \mathrm{s}$, a $2 \mathrm{~ns}$ jitter corresponding to the spatial uncertainty of about $0.2 \mathrm{~mm}$ is the limit of the spatial resolution of the system. Figure 16 shows the velocity of the plasma bullet for different frequencies with $\mathrm{O}_{2}$ percentage of $0.5 \%$ and $10 \%$. As we can see that the value of the error bar obtained from 20 repeated measurements is very small for all the experiment conditions, which means that under such conditions, the plasma propagation
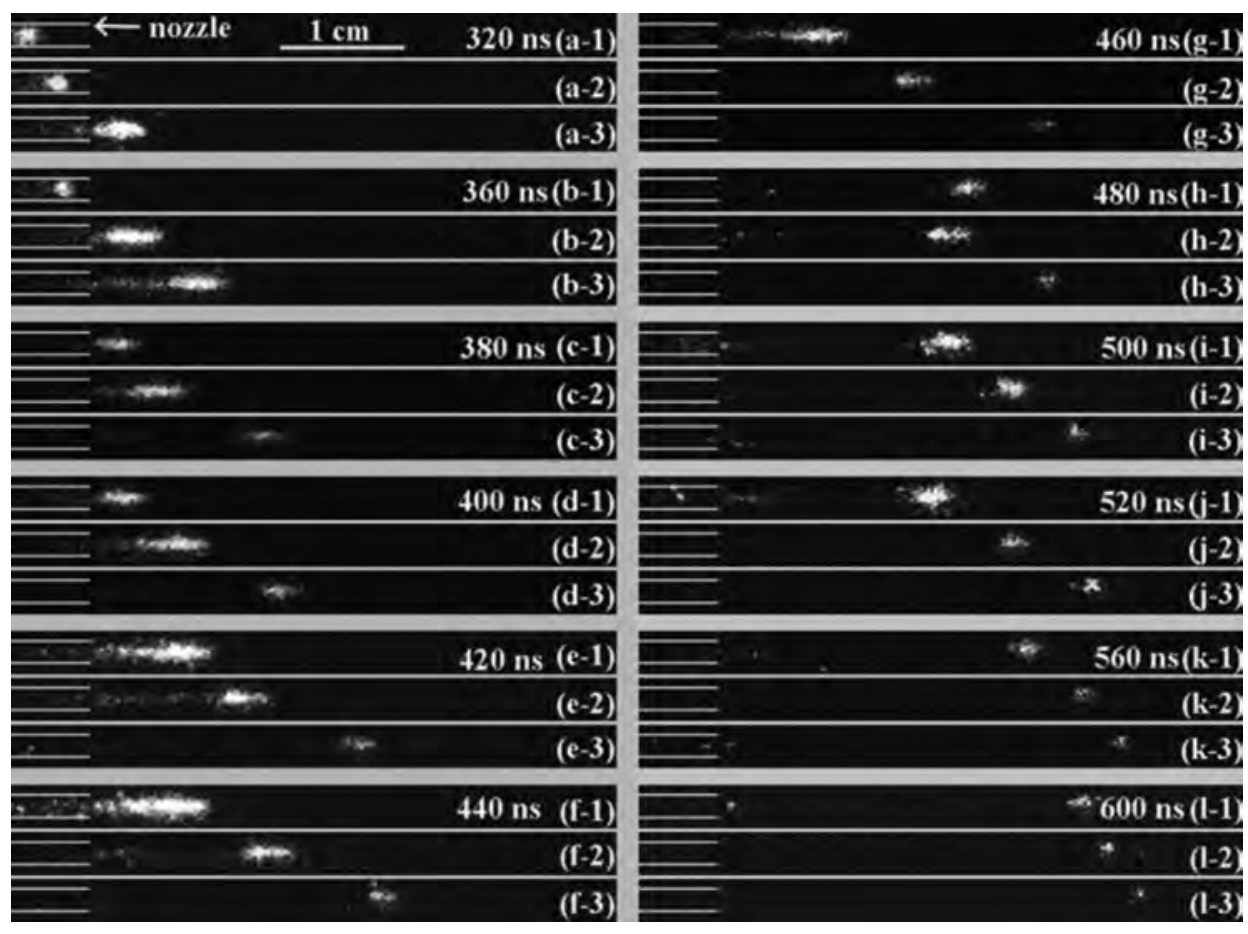

FIG. 15. High-speed photographs of the discharge with the frequency of $0.2 \mathrm{kHz}$ of the plasma plume at one atmospheric pressure plasma. Voltage: $7 \mathrm{kV}$, pulse width: $800 \mathrm{~ns}$, and helium flow rate: $11 / \mathrm{min}$. Exposure time of the ICCD camera: $20 \mathrm{~ns}$. Single shot for each image. The time labeled on the photographs corresponds to the rise edge of the applied voltage. Due to the randomness of the discharge, three photographs are taken for every delay time. ${ }^{171}$ Reproduced with permission from Wu et al., Curr. Appl. Phys. 13, S1 (2013). Copyright 2013 Elsevier. 


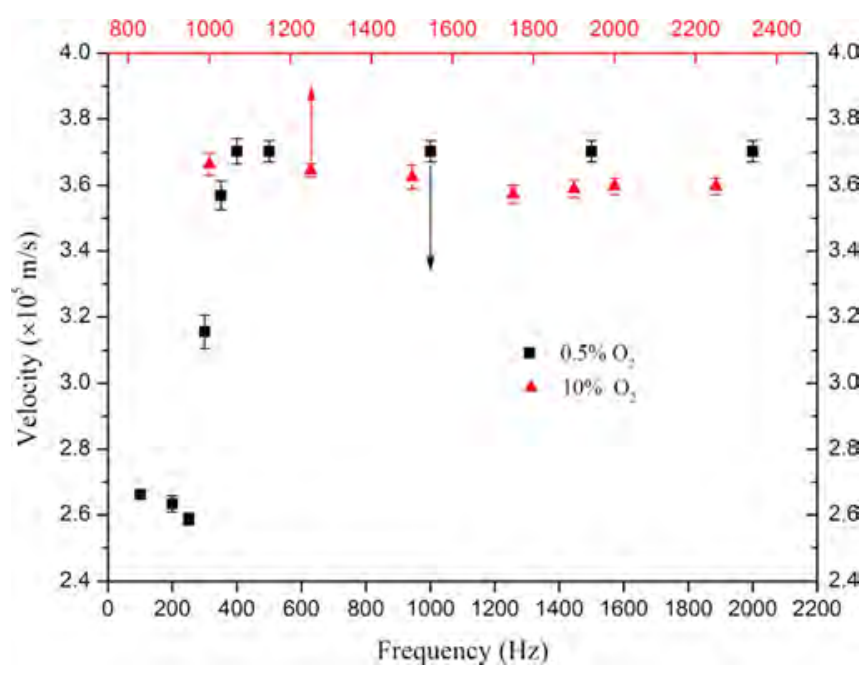

FIG. 16. The propagation velocity of the plasma bullet vs. frequency for $0.5 \%$ and $10 \%$ of $\mathrm{O}_{2}$. Total gas pressure: 0.2 atmospheric pressure. ${ }^{172}$ Reproduced with permission from Phys. Plasmas 23, 093518 (2016). Copyright 2016 AIP Publishing LLC.

velocities are constant. ${ }^{172}$ In other words, if the plasma bullet propagation is in an unrepeatable mode, then it must be due to the inconsistence of the ignition of the plasma bullet, i.e., the jitter/error bar of the ignition delay time $t_{\mathrm{d}}$.

Figure 17 shows the ignition delay time $t_{\mathrm{d}}$ for $\mathrm{O}_{2}$ percentages of $0.5 \%$ and $10 \%$ and for different frequencies. ${ }^{172}$ It can be seen from Fig. 17 that both the jitter (error bar) of delay time $t_{\mathrm{d}}$ and the delay $t_{\mathrm{d}}$ itself decrease with the increase in the frequency. As mentioned before, the plasma propagation is considered in a repeatable mode when the jitter of $t_{\mathrm{d}}$ is less than $2 \mathrm{~ns}$. The discharge mode transits from a random to a repeatable mode with the increase in the frequency. The frequency corresponding to the discharge mode transition from the random mode to the repeatable mode is defined as the critical frequency $f_{\text {cri }}$. It can be seen from Figs. 17(a) and $17(b)$, when the $\mathrm{O}_{2}$ percentages are $0.5 \%$ and $10 \%$, the corresponding critical frequencies $f_{\text {cri }}$ are 300 and $1900 \mathrm{~Hz}$, respectively.

It is worth emphasizing that although the above experiments were conducted under 0.2 atmospheric pressure, similar results were also reported under one atmospheric pressure. ${ }^{171}$ The critical frequency is about $200 \mathrm{~Hz}$ for the helium plasma jet. When the frequency is reduced to less than $200 \mathrm{~Hz}$, the plasma bullet propagation mode changes from the repeatable to the random mode.

\section{B. Propagation mode of the first discharge pulse}

For the very first discharge (ignition phase), there are no residual electrons because there is no previous discharge. In this case, the seed electron density due to natural radioactivity is very low $\left(\sim 10^{4} \mathrm{~cm}^{-3}\right)$ and the plasma bullet for the very first discharge pulse should propagate in a random mode. This was confirmed by experiment as shown in Fig. $18 .{ }^{173}$ For the very first discharge pulse, $t_{\mathrm{d}}$ is around $5 \mu \mathrm{s}$. It drops to about $300 \mathrm{~ns}$ for the second pulse and remains almost the same for the following discharge pulses. Since the jitter of $t_{\mathrm{d}}$ is much smaller than $t_{\mathrm{d}}$ itself as can been seen from Fig. 18(a), the detailed information of $t_{\mathrm{d}}$ jitter for different discharge pulses is plotted in Fig. 18(b). It clearly shows that the $t_{\mathrm{d}}$ jitter is about $1600 \mathrm{~ns}$ for the first discharge pulse; it decreases slowing to a stable value of about $3 \mathrm{~ns}$ for the 100000 th pulse.

On the other hand, a typical propagation velocity of the plasma bullet is about $10^{5} \mathrm{~m} / \mathrm{s}$. Thus, the $3 \mathrm{~ns}$ jitter corresponds to the spatial uncertainty of about $0.3 \mathrm{~mm}$, which is much less than the spatial resolution of the experiment setup. For the 100th pulse, the $t_{\mathrm{d}}$ jitter is about $7 \mathrm{~ns}$, which corresponds to the spatial uncertainly of about $0.7 \mathrm{~mm}$. This is about the limit of the spatial resolution of the system. Thus, it can be concluded that the propagation of the plasma bullets for the very first discharge is under the random mode and it becomes stable after about 100 pulses.

In addition, it is worth mentioning that the very first discharge is not ignited by the first voltage pulse that is applied to the high-voltage electrode. It is actually ignited after many voltage pulses and the number of the voltage pulses depends on the frequency of the applied voltage and on whether there is light in the room or not. ${ }^{174}$ For example, at pulse frequency of $2 \mathrm{kHz}$, when the lamp is off, the plasma is ignited at about $2000 \mathrm{~ms}$ after the voltage pulses are applied, which corresponds to 4000 voltage pulses before the plasma is ignited. On the contrary, when the lamp is on, it is decreased to less than $5 \mathrm{~ms}$, i.e., the first discharge is ignited only after less than 10 voltage pulses. Since the wavelength of light emitted by the lamp is in the range of 350 to $800 \mathrm{~nm}$ and single photon in this range cannot directly contribute to photoionization, thus the light might contribute to multiphoton ionization. The effect of visible light on the plasma jet ignition was later confirmed. ${ }^{175}$

\section{Minimum seed electron density for repeatable mode at different gas pressures}

As has been concluded above, the mode transition of plasma bullets is due to the change of the seed electron
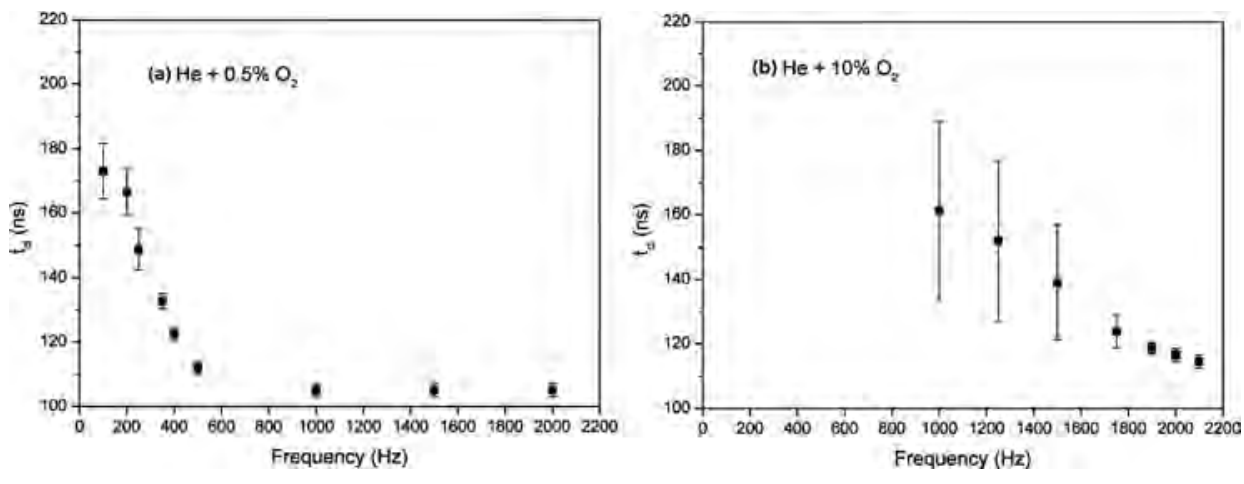

FIG. 17. $t_{\mathrm{d}}$ vs. frequencies for $\mathrm{He}$ mixed with (a) $0.5 \%$ and (b) $10 \%$ of $\mathrm{O}_{2}$. Total gas pressure: 0.2 atmospheric pressure. ${ }^{172}$ Reproduced with permission from Phys. Plasmas 23, 093518 (2016). Copyright 2016 AIP Publishing LLC. 

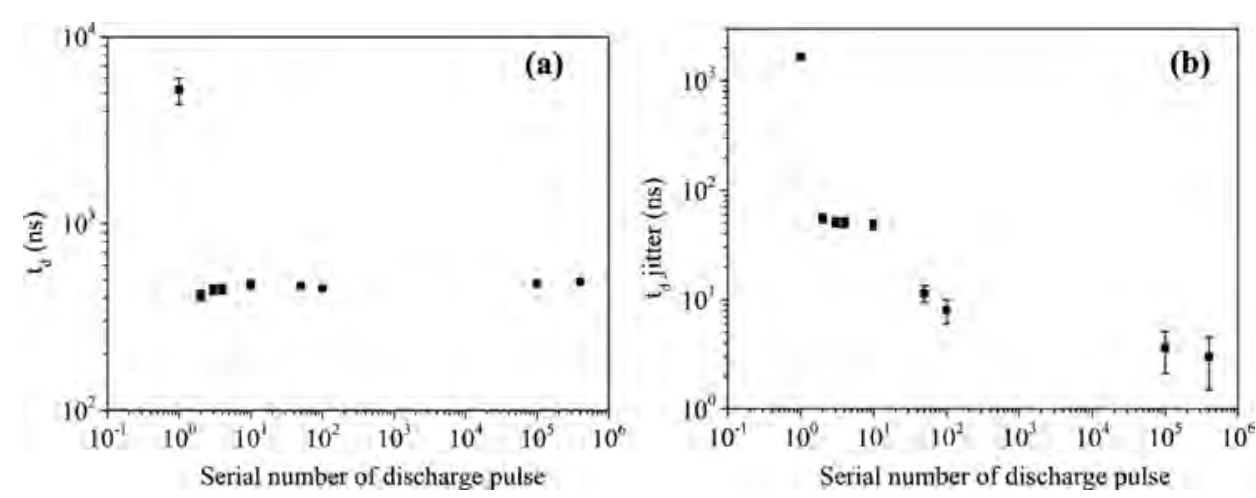

FIG. 18. The delay time $t_{\mathrm{d}}$ (a) and $t_{\mathrm{d}}$ jitter (b) for different discharge pulses. Voltage: $6 \mathrm{kV}$, pulse width: $6 \mu \mathrm{s}$, pulse frequency: $4 \mathrm{kHz}$, and helium flow rate: $11 /$ min. ${ }^{173}$ Reproduced with permission from Phys. Plasmas 21, 123509 (2014). Copyright 2014 AIP Publishing LLC. density, which results in the change of the ignition jitter of the plasma bullet. Regarding the minimum seed electron density required for $\mathrm{N}$-APPJs to propagate in a repeatable mode, it can be evaluated from the critical frequency of the mode transition. According to the measured critical frequency, the maximum decay time of the electrons to maintain the required minimum seed electron density in order to propagate in the repeatable mode can be obtained. Thus, if the initial electron density and the decay kinetics of the electrons are known, the seed electron density at the critical frequency can be evaluated.

On the other hand, the initial electron density can be estimated from the measured discharge current. For the electron decay kinetics, there are three main paths for electron decay in the afterglow, i.e., electron attachment, electron-ion recombination, and diffusion. The electron and $\mathrm{O}_{2}^{-}$diffusion coefficients scale as $D_{e}$ and $D_{\mathrm{O}_{2}^{-}} \propto 1 / P$, meaning more significant contribution of diffusion at a lower gas pressure. The decay behavior of the densities of electrons and $\mathrm{O}_{2}^{-}$can be described as follows:

$$
\begin{gathered}
\partial n_{e} / \partial t=-\left(D_{e} / \Lambda^{2}\right) n_{e}-k_{\mathrm{att}} n_{\left[\mathrm{O}_{2}\right]} n_{[\mathrm{M}]}-k_{\mathrm{rec}} n_{[\mathrm{P}]} \\
\partial n_{\mathrm{O}_{2}^{-}} / \partial t=-\left(D_{\mathrm{O}_{2}^{-}} / \Lambda^{2}\right) n_{\mathrm{O}_{2}^{-}}+k_{\mathrm{att}} n_{\left[\mathrm{O}_{2}\right]} n_{[\mathrm{M}]}-k_{\mathrm{rec}} n_{[\mathrm{P}]}
\end{gathered}
$$

where subscripts "e," "p," and "M" refer to electrons, positive ions, and neutral particles, and $\Lambda$ is the diffusion length. ${ }^{176}$ Here, $k_{\text {att }}$ is the attachment rate, $k_{\text {det }}$ is the detachment rate, and $k_{\text {rec }}$ is the recombination rate. ${ }^{177-181}$ For pure $\mathrm{He}$, the $\mathrm{O}_{2}$ concentration is too low to contribute significantly to the seed electrons. In this case, the electron density decay kinetics can be calculated approximately by Eq. (4).

When $\mathrm{O}_{2}$ is added, the critical frequency to maintain the propagation of the plasma bullet in a repeatable mode increases as discussed above. As we know, when a significant amount of $\mathrm{O}_{2}$ is added, due to attachment, electrons attach to $\mathrm{O}_{2}$ on a time scale which is much shorter than the pulse off time, and the detachment of $\mathrm{O}_{2}^{-}$besides the residual electron left over from previous discharges could contribute significantly to the seed electron density. This is because the electric field near the tip of the high voltage electrode and also at the plasma bullet head can be very high, ${ }^{182}$ thus resulting in a significant electron detachment from $\mathrm{O}_{2}^{-}$. Importantly, electron detachment is the main source of the seed electrons when a significant amount of $\mathrm{O}_{2}$ is added. The electron density due to detachment $n_{\text {det }}$ can be calculated from the following equation:

$$
\partial n_{\mathrm{det}} / \partial t=k_{\mathrm{det}} n_{\left[\mathrm{O}_{2}\right]} n_{[\mathrm{M}]}
$$

where $k_{\text {det }}$ is the detachment coefficient. On the other hand, it should be emphasized that $k_{\operatorname{det}}$ strongly depends on the value of the reduced electric field $E / N$. Therefore, electron detachment from $\mathrm{O}_{2}^{-}$contributes significantly to the seed electron density only when the electric field is sufficiently high.

In order to have significant electron detachment, ${ }^{113}$ the value of $E / N$ should be higher than $150 \mathrm{Td}$. The period while the reduced electric field is higher than 150 Td can be estimated according to the pulse rising time, the ignition delay time, and the electrode configuration. ${ }^{172}$

Now, according to Eqs. (3)-(5), as long as the initial electron density is given, the decay kinetics of the electron density can be obtained according to a simple zero-dimensional kinetic model. For the helium atmospheric pressure plasma jet, ${ }^{171}$ although the initial electron density is not known, however, the initial electron density of a helium plasma jet should be in the range of $10^{11} \mathrm{~cm}^{-3}-10^{13} \mathrm{~cm}^{-3} \cdot{ }^{183,184}$ Therefore, assuming that the initial electron densities are $10^{11}, 10^{12}$, and $10^{13} \mathrm{~cm}^{-3}$, respectively, the corresponding decay behaviors of the seed electron density are calculated as shown in Fig. 19. The propagation mode transition of the helium plasma jet has a critical frequency of $250 \mathrm{~Hz}$, which corresponds to the decay time of 4 ms. Thus, according to Fig. 19, the seed electron density of the helium plasma jet to propagate in a repeatable mode is about $10^{9}$ to $1.3 \times 10^{9} \mathrm{~cm}^{-3}$ when the initial electron density varies from $10^{11}$ to $10^{13} \mathrm{~cm}^{-3}$. Fortunately, the critical seed electron density is not sensitive to the initial electron density.

In order to know whether the minimum seed electron density required for a repeatable mode is strongly gas composition dependent or not, the ideal choice will be to generate plasma plumes at one atmospheric pressure with different working gas mixtures and then to obtain their initial electron density and the critical frequency of mode transitions. Finally, based on the obtained results and Eqs. (1)-(3), the minimum electron density required for the repeatable mode can be calculated for different gas composition. However, to generate atmospheric pressure plasma jet for different gas composition such as air, much higher voltages are needed; however, the power supplies of this kind are not commonly available or very expensive to custom-build. A compromise 


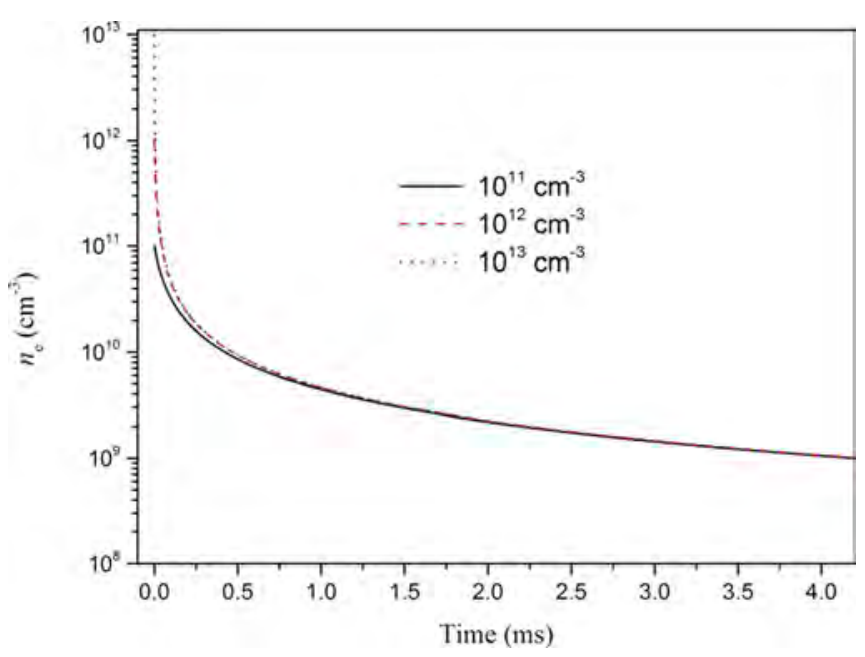

FIG. 19. The calculated curves of the electron density decay at atmospheric pressure with the initial electron density of $10^{11}, 10^{12}$, and $10^{13} \mathrm{~cm}^{-3} .^{172}$ Reproduced with permission from Phys. Plasmas 23, 093518 (2016). Copyright 2016 AIP Publishing LLC.

could be achieved by running experiments at a reduced gas pressure of 0.2 and 0.04 atmospheric pressure; the minimum seed concentrations for a repeatable mode are calculated for different gas mixtures as shown in Figs. 20 and 21.

Figures 20 and 21 show that the minimum seed electron number density does not strongly depend on the oxygen concentration; they are on the order of $10^{8} \mathrm{~cm}^{-3}$ for 0.2 atmospheric pressure and $10^{7} \mathrm{~cm}^{-3}$ for 0.04 atmospheric pressure. $^{185}$ Besides, simulation results show that, at 0.2 atmospheric pressure, for the case of helium, the residual electrons left over from previous discharges are about $10^{8} \mathrm{~cm}^{-3}$, which contributes most of the seed electrons. When a small $(\sim 1 \%)$ amount of $\mathrm{O}_{2}$ is added, the residual electron density is below $10^{6} \mathrm{~cm}^{-3}$ for a pulse frequency of $10 \mathrm{kHz}$ as shown in Fig. 22, ${ }^{185}$ which is two orders of magnitude lower than the required seed electron density for the plasma to propagate in the repeatable mode. The seed electrons due to detachment from $\mathrm{O}_{2}{ }^{-}$become the main source. This is also true for the case of 0.04 atmospheric pressure.

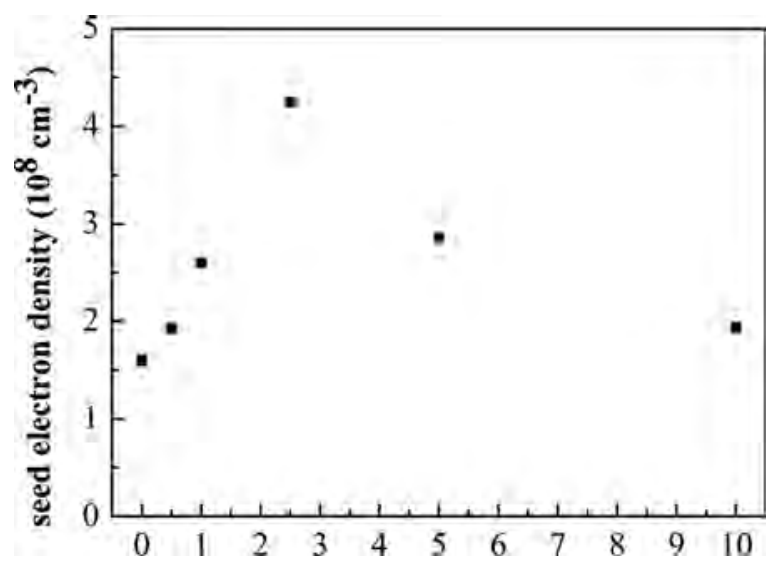

FIG. 20. The required seed electron density $n_{\text {seed }}$ for the plasma to propagate in a repeatable mode at 0.2 atmospheric pressure with helium mixed with $\mathrm{O}_{2}$ fractions of $0 \%, 0.5 \%, 1 \%, 2.5 \%, 5 \%$, and $10 \%$. Air impurity of $0.025 \%$ is always present. ${ }^{185}$ Reproduced with permission from Phys. Plasmas 23, 123513 (2016). Copyright 2016 AIP Publishing LLC.

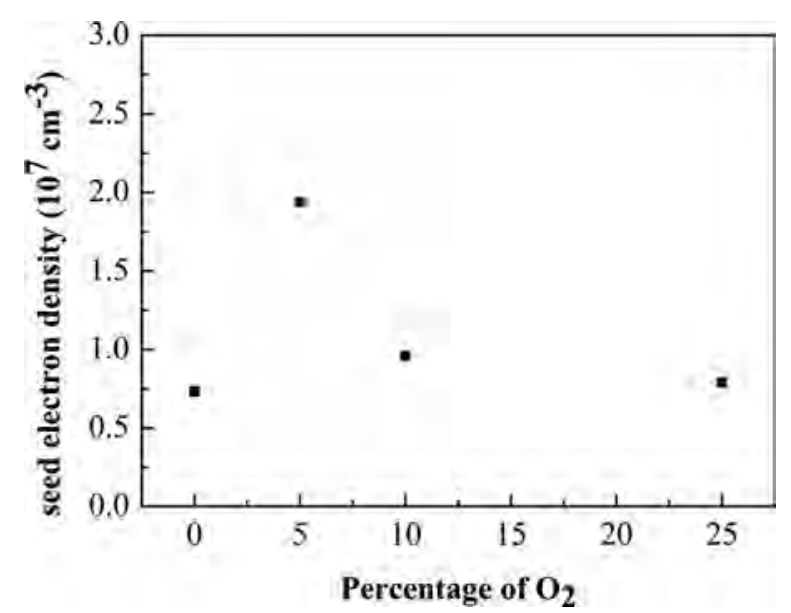

FIG. 21. The required seed electron density $n_{\text {seed }}$ for the plasma to propagate in a repeatable mode at 0.04 atmospheric pressure with $\mathrm{O}_{2}$ fraction of 0,5 , 10 , and 25 in $\mathrm{He} / \mathrm{O}_{2}$. Air impurity of $0.025 \%$ is always present. ${ }^{185}$ Reproduced with permission from Phys. Plasmas 23, 123513 (2016). Copyright 2016 AIP Publishing LLC.

Furthermore, a traditional streamer evolves from an avalanche if the electric field $E^{\prime}$ induced from the space charge in the streamer head becomes comparable to the external field $E_{0}{ }^{29}$ Since plasma bullets are also a kind of streamer discharges, plasma bullets should also meet this criterion, taking into account the difference in the seed electron density between these two discharges. To validate this assumption, according to the estimated $n_{\text {seed, }}$, the space charges resulting from the avalanche at a distance $l_{a v}$ from the anode were calculated, and the electric field induced by the space charge is calculated. It was found that, for a gas pressure of $2 \times 10^{4} \mathrm{~Pa}$, the local electric field $E^{\prime}$ induced by the space charge is $13.1 \mathrm{kV} / \mathrm{cm}$. Meanwhile, the electric field $E_{0}$ due to the applied voltage is $15.8 \mathrm{kV} / \mathrm{cm}$, which is quite close to the local electric field $E^{\prime}$ induced by the space charge. Similarly, for a gas pressure of $4 \times 10^{3} \mathrm{~Pa}$, the electric field induced by the space charge is $19.2 \mathrm{kV} / \mathrm{cm}$, while the value of $E_{0}$ is $19.2 \mathrm{kV} / \mathrm{cm}$. These values are remarkably the same. Thus, as the seed electron density meets the critical number, the streamer will ignite at a definite time, which means that the discharge propagates in the repeatable mode. However, if the seed electron density is lower than the critical value, more time is needed for the avalanche to develop until the electric field of the space charge satisfies the criterion of streamer formation. In this case, the discharge propagates in the random mode.

\section{Photoionization for plasma bullet}

Regarding the role of photoionization in the propagation of plasma bullets, again, because of the major experimental difficulties, there are no direct photoionization experiments reported. For simplicity, background ionization was used to replace photoionization in most of the simulations. The seed electron densities were assumed by varying from $10^{2}$ to $10^{10} \mathrm{~cm}^{-3} \cdot{ }^{186-198}$ It is worth emphasizing that, in the simulations, ${ }^{194}$ photoionization was not included, if the initial electron density $n_{0}$ is assumed to be lower than $10^{9} \mathrm{~cm}^{-3}$; the development of the plasma is restricted to a zone inside the 

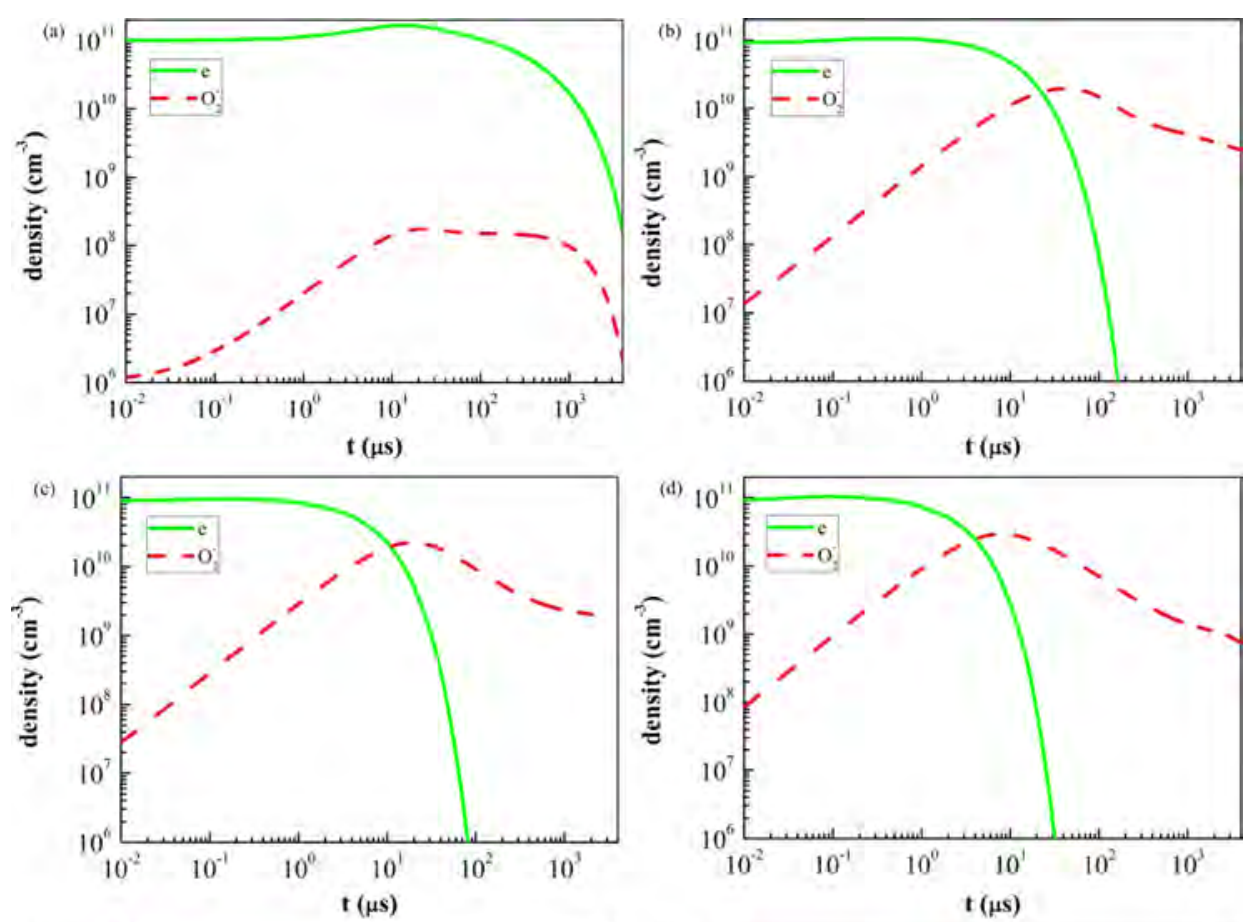

FIG. 22. The temporal evolution of $\mathrm{O}_{2}{ }^{-}$ions and electrons in the afterglow (no applied voltage) at a total pressure of $2 \times 10^{4} \mathrm{~Pa}$ for $\mathrm{O}_{2}$ percentages of (a) $0 \%$, (b) $0.5 \%$, (c) $1 \%$, (d) $2.5 \%$, (e) $5 \%$, and (f) $10 \%$. Air impurity of $0.025 \%$ is always present. ${ }^{185}$ Reproduced with permission from Phys. Plasmas 23, 123513 (2016). Copyright 2016 AIP Publishing LLC.
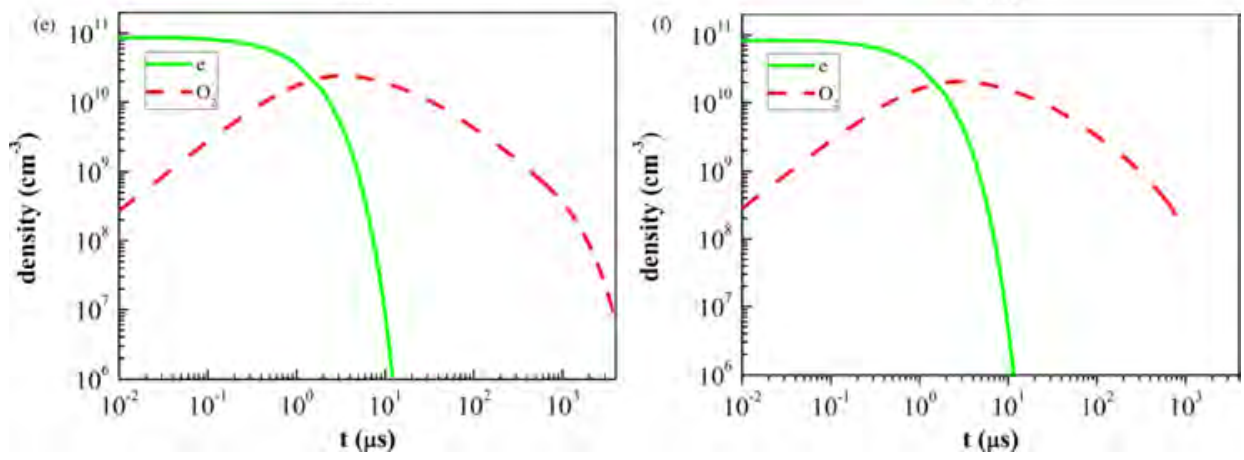

tube. The plasma cannot propagate in the open air when the initial electron density is assumed to be lower than $10^{9} \mathrm{~cm}^{-3}$.

In order to better understand the role of photoionization in the propagation of plasma bullets, photoionization effect is incorporated in the modeling. ${ }^{182,186}$ Two simulations were carried out, one with photoionization and another one without photoionization. The seed electron density in these simulations was taken equal to $10^{3} \mathrm{~cm}^{-3}$. Calculations of the total electron ionization rate as a function of time for the two cases revealed that basic phenomena of streamer propagation along the jet axial direction appear to be quite similar for the both cases. ${ }^{182}$ It was concluded that streamer propagation in cold plasma jets is possible at such low $n_{0}$ even without photoionization, although it is much slower compared to the case when photoionization is included in the model. Indeed, photoionization processes create a weakly ionized plasma cloud $\left(\sim 10^{6}-10^{8} \mathrm{~cm}^{-3}\right)$ surrounding the primary ionization processes in the streamer head. ${ }^{186}$ This cloud is responsible for providing seed electrons that significantly increase the streamer propagation speed. We emphasize that these calculations are based on the fluid models. As mentioned above, these models cannot adequately describe the experimentally observed ignition delay and its jitter. Further complications may be expected upon transition into microplasma domain. $^{199,200}$

\section{PHYSICAL MECHANISMS}

\section{A. Minimum SED for repeatable propagation mode in pure helium plasma}

As discussed in Sec. IV, the plasma propagation behavior for the very first discharge is always in a stochastic mode and it transits to a repeatable mode after about 100 discharge pulses. However, the available plasma jet models consider only one single discharge without accounting for charge accumulation effects.

We recall that the accumulation effects include the accumulation of electrons, ions, metastable excited states, and other reactive species. The accumulation of free electrons is normally approximated by the initial electron density. If the working gas is pure helium, the main charged species would be electrons, $\mathrm{He}^{+}$, and $\mathrm{He}_{2}{ }^{+}$, according to

$$
\begin{gathered}
\mathrm{He}^{+}+\mathrm{He}+\mathrm{He} \rightarrow \mathrm{He}_{2}^{+}+\mathrm{He}, \\
\left(k_{1}=6.6 \times 10^{-32} \mathrm{~cm}^{-6} \times \mathrm{s}^{-1}\right) .
\end{gathered}
$$

The exponential delay time of the process at atmospheric pressure is $t=\left(\mathrm{k}[\mathrm{He}]^{2}\right)^{-1}=1.5 \times 10^{-8} \mathrm{~s}$, which means that the main ions will be $\mathrm{He}_{2}{ }^{+}$since recombination of electrons and $\mathrm{He}^{+}$will take much longer time during the decay period when the electron density is $\sim 10^{10} \mathrm{~cm}^{-3}$ or lower. At 
atmospheric pressure, ignoring the diffusion loss, the following reactions describe the transformations of He species: ${ }^{201}$

$$
\begin{gathered}
\mathrm{He}_{2}^{+}+\mathrm{e}+\mathrm{He} \rightarrow \mathrm{He}+\mathrm{He}+\mathrm{He}, \\
\left(k_{2}=2.0 \times 10^{-27} T_{e g}^{-2.5} \mathrm{~cm}^{-6} \cdot \mathrm{s}^{-1}\right), \\
\mathrm{He}_{2}^{+}+\mathrm{e}+\mathrm{He} \rightarrow \mathrm{He}+\mathrm{He}+\mathrm{He}^{*}, \\
\left(k_{3}=5.0 \times 10^{-27} T_{e g}^{-1.0} \mathrm{~cm}^{-6} \cdot \mathrm{s}^{-1}\right), \\
\mathrm{He}_{2}^{+}+\mathrm{e}+\mathrm{e} \rightarrow \mathrm{He}+\mathrm{He}+\mathrm{e}, \\
\left(k_{4}=7.0 \times 10^{-20}\left(300 / \mathrm{T}_{\mathrm{e}}\right)^{4.5} \mathrm{~cm}^{-6} \cdot \mathrm{s}^{-1}\right), \\
\mathrm{He}_{2}^{+}+\mathrm{e}+\mathrm{e} \rightarrow \mathrm{He}+\mathrm{He}^{*}+\mathrm{e}, \\
\left(k_{5}=1.0 \times 10^{-20} T_{e g}^{-4.0} \mathrm{~cm}^{-6} \cdot \mathrm{s}^{-1}\right), \\
\mathrm{He}_{2}^{+}+\mathrm{e} \rightarrow \mathrm{He}^{+} \mathrm{He}, \quad\left(k_{6}=8.9 \times 10^{-9} T_{e g}^{-1.5} \mathrm{~cm}^{-3} \cdot \mathrm{s}^{-1}\right) \\
\mathrm{He}_{2}^{+}+\mathrm{e} \rightarrow \mathrm{He}+\mathrm{He}, \quad\left(k_{7}=1.0 \times 10^{-8} \mathrm{~cm}^{-3} \cdot \mathrm{s}^{-1}\right) .
\end{gathered}
$$

The exponential decay time depends on the corresponding electron density at the moment. Besides, during the decay phase, there is no external electric field, it is reasonable to assume $T_{\mathrm{e}}=T_{\mathrm{g}}=300 \mathrm{~K}$. Immediately after the discharge, the electron density is on the order of $10^{11}-10^{13} \mathrm{~cm}^{-3}$. Thus, in the following estimate, it is assumed to be $10^{12} \mathrm{~cm}^{-3}$, so according to reactions (7)-(12), the decay time constant is about $3.6 \mu$ s. On the other hand, the critical electron density for propagation mode transition is around $10^{9} \mathrm{~cm}^{-3}$. Thus, for the electron density of $10^{9} \mathrm{~cm}^{-3}$, the corresponding time constant can also be estimated according to reactions (7)-(12); it is about $5 \mathrm{~ms}$, which corresponds to frequency of $200 \mathrm{~Hz}$, consistently with the experimental studies of plasma plume repeatability. ${ }^{172}$

\section{B. Minimum SED for repeatable propagation mode with $\mathrm{O}_{2}$ present}

\section{SED due to $\mathrm{O}_{2}^{-}$detachment}

For the plasma jet to propagate in open air, because of diffusion of surrounding air, $\mathrm{O}_{2}$ density in the plasma plume could be high, thus $\mathrm{O}_{2}^{-}$concentration could also be high. So, the seed electrons could be produced by detachment. The formation of $\mathrm{O}_{2}{ }^{-}$is described by the attachment pathways $^{201,202}$

$$
\begin{aligned}
& \mathrm{e}+\mathrm{O}_{2}+\mathrm{O}_{2} \rightarrow \mathrm{O}_{2}^{-}+\mathrm{O}_{2}, \quad k_{8}=2.26 \times 10^{-30} \mathrm{~cm}^{6} \mathrm{~s}^{-1} \\
& \mathrm{e}+\mathrm{O}_{2}+\mathrm{N}_{2} \rightarrow \mathrm{O}_{2}^{-}+\mathrm{N}_{2}, \quad k_{9}=1.24 \times 10^{-31} \mathrm{~cm}^{6} \mathrm{~s}^{-1}
\end{aligned}
$$

The exponential decay time of the electron $\Delta t=\left(k_{8}\left[\mathrm{O}_{2}\right]^{2}\right.$ $\left.+k_{9}\left[\mathrm{O}_{2}\right]\left[\mathrm{N}_{2}\right]\right)^{-1}$. At room temperature, $k_{8}=2.26 \times 10^{-30}$ $\mathrm{cm}^{6} \mathrm{~s}^{-1}$ and $k_{9}=1.24 \times 10^{-31} \mathrm{~cm}^{6} \mathrm{~s}^{-1} \cdot 36,39$ At atmospheric pressure air conditions, it is about $20 \mathrm{~ns}$. After forming $\mathrm{O}_{2}{ }^{-}$, if the repetition frequency is high enough, before $\mathrm{O}_{2}{ }^{-}$disappear through two and three-body ion-ion recombination or diffusion, these species can produce seed electrons through detachment when the next voltage pulse is applied.
For example, for $1 \% \mathrm{O}_{2}$ in helium at 0.2 atmospheric pressure, the $\mathrm{O}_{2}{ }^{-}$density is about $2 \times 10^{9} \mathrm{~cm}^{-3}$ for a pulse frequency of $400 \mathrm{~Hz}$, which is the critical frequency for the repeatable propagation mode. ${ }^{172,185}$ The seed electrons density due to the detachment is about $2.5 \times 10^{8} \mathrm{~cm}^{-3}$ while the original leftover free electron density is much lower than $10^{6} \mathrm{~cm}^{-3} \cdot{ }^{185}$ In other words, electron detachment is the main contribution of seed electrons when $\mathrm{O}_{2}$ is present.

Another study shows that, at atmospheric pressure, the critical frequency for the repeatable propagation mode is about $250 \mathrm{~Hz}$, while the density of leftover free electrons from previous pulses is around $10^{4}-10^{5} \mathrm{~cm}^{-3}$. ${ }^{171}$ In this case, the density of electrons detached from $\mathrm{O}_{2}{ }^{-}$to produce most of the seed electrons should be about $10^{9}$ to $1.3 \times 10^{9} \mathrm{~cm}^{-3}$, while the initial electron density varies from $10^{11}$ to $10^{13} \mathrm{~cm}^{-3} \cdot{ }^{172}$ It is worth pointing out that the critical frequency for the propagation mode transition of the plasma bullet depends on the $\mathrm{O}_{2}$ percentage. Further estimates show that the minimum seed electron density is about the same for all different percentages of $\mathrm{O}_{2}$ and the helium mixture. However, this value strongly depends on the gas pressure. Specifically, it is about $10^{7} \mathrm{~cm}^{-3}$ for a gas pressure of $4 \mathrm{kPa}$, $10^{8} \mathrm{~cm}^{-3}$ for a gas pressure of 0.2 atmospheric pressure, and $10^{9} \mathrm{~cm}^{-3}$ for one atmospheric pressure.

\section{SED due to photoionization}

On the other hand, photoionization is always present because there is a trace amount of air in the plasma jet when it propagates in surrounding air. For a positive streamer in air, it is believed that photoionization is essential for propagation of the streamer. At one atmospheric pressure, the electron density induced by photoionization is on the order of $10^{7} \mathrm{~cm}^{-3}$ because photoionization affects the propagation of the streamer only when the seed electron density is lower than $10^{7} \mathrm{~cm}^{-3} \cdot{ }^{94}$ Numerical modeling ${ }^{124}$ also indicates that the electron density induced by photoionization is on the order of $10^{7} \mathrm{~cm}^{-3}$ because similar streamer propagation behavior can be obtained in the model where either only seed electron density is $10^{7} \mathrm{~cm}^{-3}$ is assumed or only photoionization is included. Another study ${ }^{117}$ shows that $\mathrm{O}_{2}{ }^{-}$ density of $10^{9} \mathrm{~cm}^{-3}$ has a similar contribution as photoionization, since not all $\mathrm{O}_{2}{ }^{-}$lost their electron during the propagation of the plasma bullet. One can thus conclude that the maximum electron density contributed by photoionization should be less than $10^{9} \mathrm{~cm}^{-3}$.

However, results of other authors introduce even more uncertainty into the real potential of photoionization to generate seed electrons for the guided streamer propagation. It was reported ${ }^{185}$ that for $\mathrm{O}_{2}{ }^{-}$density of $2 \times 10^{9} \mathrm{~cm}^{-3}$, the electron density due to detachment is almost one order of magnitude lower, i.e., $2.5 \times 10^{8} \mathrm{~cm}^{-3}$. In other words, only about $10 \%$ of the negative $\mathrm{O}_{2}{ }^{-}$ions have lost their electron when the high electric field was applied. Therefore, it is reasonable to assume that the electron density induced by photoionization is on the order of $10^{8} \mathrm{~cm}^{-3}$. This value is consistent with the numerical results suggesting that a weakly ionized plasma cloud induced by photoionization and surrounding the streamer head has the electron density 
of up to $10^{8} \mathrm{~cm}^{-3}$, while this density naturally decreases with the increase in the distance from the streamer head. ${ }^{182,186}$ Another important conclusion of these works is that the photoionization could induce a significant amount of seed electrons at the distance up to about $2 \mathrm{~mm}$. One more study shows that when $\mathrm{O}_{2}{ }^{-}$concentration is below $10^{9} \mathrm{~cm}^{-3}$, the electron detachment mechanism appears to be less important than photoionization. ${ }^{84}$ Since not all negative $\mathrm{O}_{2}{ }^{-}$ ions lose electron during the propagation time of about $4 \mathrm{~ns}$, the seed electron density due to detachment should be also less than $10^{9} \mathrm{~cm}^{-3}$.

Based on all these studies, it can be concluded that the density of electrons realistically produced by photoionization is on the order of $10^{7}-10^{8} \mathrm{~cm}^{-3}$. It should be emphasized that the electrode configuration, the applied voltage, and the gas composition in these studies are different, but the obtained electron density induced by photoionization is not too far away from each other.

\section{Required minimum SED for repeatable propagation mode}

According to the discussion above, for the case of pure helium plasmas, the minimum required SED for a repeatable propagation mode at atmospheric pressure is on the order of $10^{9} \mathrm{~cm}^{-3}$. When $\mathrm{O}_{2}$ is present, electrons attach to $\mathrm{O}_{2}$ molecules in about $20 \mathrm{~ns}$ and form negative $\mathrm{O}_{2}{ }^{-}$ions. For repetitive discharges, if the repetition frequency is high enough, the negative $\mathrm{O}_{2}{ }^{-}$ions could contribute significantly to the concentration of free electrons. Although different $\mathrm{O}_{2}$ percentage in helium results in different critical frequency to maintain the repeatable mode, it was found that the minimum SED for the repeatable mode propagation is the same as for the pure helium case, which is also $10^{9} \mathrm{~cm}^{-3}$ for atmospheric pressure plasma jets. Further investigation shows that electron density induced by photoionization is at least one to two orders less than the required minimum SED for repeatable mode propagation. This means that photoionization alone is not able to meet the requirement of having plasma to propagate under repeatable mode.

On the other hand, when the gas pressure is reduced, the required minimum SED for the repeatable mode propagation also decreases. For a gas pressure of 0.2 and 0.04 atmospheric pressure, the corresponding required minimum SED for the repeatable mode propagation is on the order of $10^{8}$ and $10^{7} \mathrm{~cm}^{-3}$.

\section{FUTURE CHALLENGES AND OPPORTUNITIES}

\section{A. Minimum seed electron density for air streamer propagating in repeatable mode}

For traditional streamers, one experiment showed that when the seed electron density is on the order of $10^{5} \mathrm{~cm}^{-3}$, it starts affecting the branching behavior, i.e., the spatial repeatability of the streamer. ${ }^{87}$ Another experimental study found that a streamer becomes diffused when the seed electron density is on the order of $\sim 10^{7} \mathrm{~cm}^{-3}{ }^{88}$ Simulations of streamers in air show that the electron density from the photoionization could reach $\sim 10^{8} \mathrm{~cm}^{-3} \cdot{ }^{94,119}$ However, to the best of our knowledge, streamer propagating in air in the repeatable mode is yet to be observed. Thus, for traditional streamers to propagate in the repeatable mode, the seed electron density must be higher than $\sim 10^{8} \mathrm{~cm}^{-3}$.

Besides, simulation results show that when the seed electron density is $\sim 10^{9} \mathrm{~cm}^{-3}$, the streamer starts propagating instantly. On the country, as the seed electron density decreases, the delay for the streamer formation starts increasing. ${ }^{94}$ However, this conclusion was based on the fluid model, which cannot describe the jitter of delay, as discussed previously. In other words, it is impossible to simulate the mode transition behavior because of the nature of the model. However, such results indicate that electron density of $10^{9} \mathrm{~cm}^{-3}$ might be a threshold for the air streamer to propagate differently. Nevertheless, in order to confirm whether the minimum seed electron density rule applies to traditional air streamers, further experiments and simulations are needed.

\section{B. Stabilization period of repeatable propagation mode of plasma bullet}

Besides accumulation of seed electrons, the accumulation of excited metastable states, other reactive species generated by the plasma should also be simulated because they could also be either directly ionized or indirectly affect the final concentration of positive and negative ions and thus affect the seed electrons concentration in the stable state. For example, if a significant amount of $\mathrm{O}_{2}$ is in the excited metastable states, then there will also be a significant amount of $\mathrm{O}_{2}{ }^{-}$in excited metastable states. In this case, one could expect electron detachment from excited metastable negative $\mathrm{O}_{2}{ }^{-}$ions to be significantly higher compared to the ground state $\mathrm{O}_{2}^{-}$.

Importantly, the accumulation effect was confirmed by experiment. ${ }^{173}$ It was found that the very first discharge always propagates in a stochastic mode. It transfers to a repeatable mode after about 100 pulses for a frequency of $4 \mathrm{kHz}$, which corresponds to about $25 \mathrm{~ms}$. This means that some species accumulate to reach a stable concentration and they are able to ensure sufficiently high SED after a relatively long period of time. However, the exact nature and abundance of the species involved are not clearly understood at the moment and further research is needed. Such studies should measure the absolute concentrations of the relevant species, which could be a significant technical challenge. On the other hand, numerical modeling is promising to provide more insights on these species and the associated mechanisms of charge generation and accumulation.

\section{Detachment rate of $\mathrm{O}_{2}{ }^{-}$}

As mentioned above, at room temperature, in order to have electrons detached from $\mathrm{O}_{2}^{-}$, a high electric field is needed. This is because, when high electric field is present, ions gain energy and increase their effective temperature, which results in the enhanced detachment of electrons.

Figure 23 compares the detachment rate constant from simulations and experimental measurements in oxygen gas at $T=300 \mathrm{~K}$. There is a good agreement between the simulation, ${ }^{202}$ semi-empirical calculation, ${ }^{203}$ and experiment. ${ }^{204}$ 


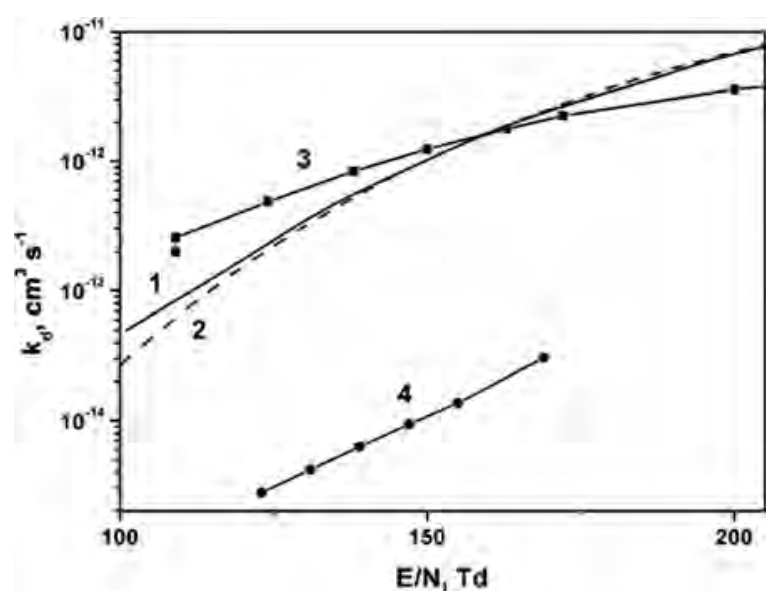

FIG. 23. The detachment rate constant as a function of reduced electric field in oxygen at $T=300 \mathrm{~K}$. Curve 1 represents the results of numerical simulations, curve 2 corresponds to a semi-empirical calculation, and curves 3 and 4 show the results of experimental measurements. ${ }^{202}$ Reproduced with permission from N. Aleksandrov and E. Anokhin, J. Phys. B: At., Mol., Opt. Phys. 44, 115202 (2011). Copyright 2011 Institute of Physics.

However, the difference between the calculations and the measurements (curve 4) is very large, up to two orders of magnitude. $^{205}$ It is worth pointing out that electron detachment rates are also very sensitive to the gas temperature. Further studies are needed to obtain more reliable detachment rates of $\mathrm{O}_{2}{ }^{-}$under the varied reduced electric field and gas temperature. As shown in Fig. 24, in the region of the reduced electric field between 50 and $100 \mathrm{Td}$, the detachment rate constant increase about one order of magnitude or even more when the gas temperature increase by $200 \mathrm{~K}$.

At room temperature atmospheric pressure $\mathrm{O}_{2}$ condition, according to Fig. 24, for electric field of $150 \mathrm{Td}$, the detachment rate constant is on the order of $10^{-12} \mathrm{~cm}^{3} \mathrm{~s}^{-1}$. Thus, the exponential decay time of the detachment is $\Delta t$ $=\left(k_{\mathrm{det}} \cdot\left[\mathrm{O}_{2}\right]\right)^{-1} \approx 40 \mathrm{~ns}$. Thus, in order to have a significant amount of $\mathrm{O}_{2}{ }^{-}$detached, the strength of the electric field, the rising time, and the peak shape of the applied voltage are crucial for determining the seed electron concentration and the propagation mode. Further investigations are also urgently needed.

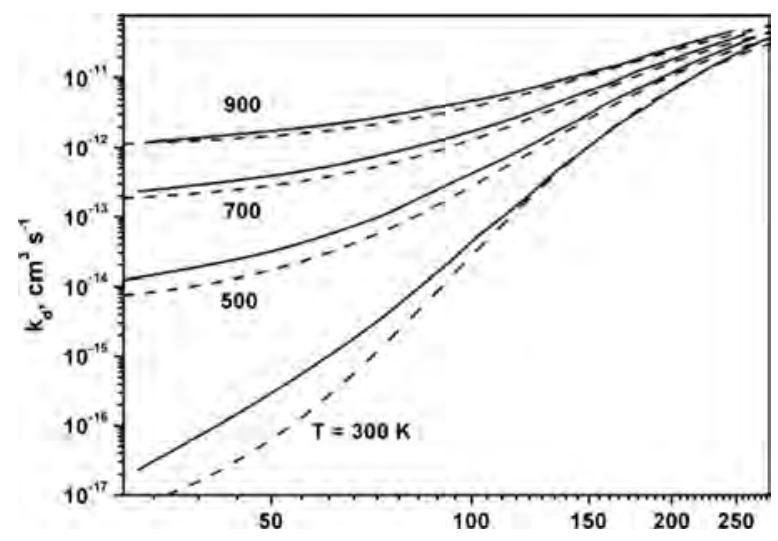

FIG. 24. The detachment rate constant as a function of the reduced electric field in oxygen at various gas temperatures $T$. The solid curves correspond to numerical simulations and the dashed curves correspond to a semiempirical calculation. ${ }^{202}$ Reproduced with permission from N. Aleksandrov and E. Anokhin, J. Phys. B: At., Mol., Opt. Phys. 44, 115202 (2011). Copyright 2011 Institute of Physics.

\section{Photoionization models}

Current photoionization models assume that excited $\mathrm{N}_{2}$ emits radiation in the range of $98-102.5 \mathrm{~nm}$, which can be absorbed by the $\mathrm{O}_{2}$ species and cause ionization. However, experiments ${ }^{117,118}$ found that streamer propagation behavior does not change much when $\mathrm{O}_{2}$ concentration is varied significantly. In the experiment where $\mathrm{N}_{2}+\mathrm{O}_{2}$ mixture was used, when $\mathrm{O}_{2}$ concentration was varied from less than 1 ppm to six orders of magnitude higher, the streamer propagated with nearly the same velocity. This interesting observation is not clearly understood at the moment. Indeed, according to the current photoionization model, if photoionization is important in the streamer propagation, variation of the $\mathrm{O}_{2}$ density should definitely affect it.

This intriguing discrepancy causes one to question the currently widely accepted photoionization pathways. One possibility may be related to multi-photon ionization, especially two-photon ionization. The second potential pathway may be due to stepwise ionization where the ionized molecules/atoms find themselves in excited states rather than in the ground state. The latter mechanism is unlikely to dominate during the very first discharge. However, it is possible for the following discharges especially when the frequency of the discharge is relatively high and since some of the metastable states of $\mathrm{N}_{2}$ and $\mathrm{O}_{2}$ have relatively long lifetimes.

\section{E. Streamer simulation models}

Most of the streamer simulations reported use a classical fluid model, which cannot reproduce the branching and the stochastic behavior of the streamers. In order to better understand the plasma bullet behavior, fluid models should be enhanced with specific features to reflect the nature of the streamer behavior. A potential solution could be sought through the development of hybrid models. One such hybrid model incorporates statistical photon transport effects into a fluid-based plasma transport model. This hybrid model is able to describe random photoionization phenomena and thus the branching behavior of streamers. ${ }^{91}$ In this model, an initial plasma cloud surrounding the central powered electrode with a radius of $1.5 \mathrm{~mm}$ and peak value of $n_{\mathrm{e}}=10^{10} \mathrm{~cm}^{-3}$ is assumed. Another hybrid model uses a Monte Carlo collision - particle in cell method (PIC-MCC) to describe the motion and collisions of free electrons in a streamer discharge in the region of high fields without photoionization and low electron densities with a fluid model in the rest of the domain. This approach was used to simulate the branching behavior, and the results were compared with the particle model based on the PIC-MCC method. ${ }^{82}$ In the both hybrid and particle models, the particle model with a single electron is used in the early stages, until the number of electrons reaches $2 \times 10^{7}$; this occurs at about $0.46 \mathrm{~ns}$. At this time, the particle model switches to the super-particle mode, while the hybrid model switches to the full hybrid scheme. However, even these hybrid models still cannot simulate the behavior of the ignition delay and its jitter. Therefore, further development of new advanced models to simulate the mode transition behavior of plasma bullets between the repeatable and stochastic mode is on the agenda. 


\section{F. New phenomena in plasma plumes}

One of the relatively newly reported and the as yet unexplained phenomena in plasma jets is the segmented structure of the plasma plume depicted in Fig. $4 .^{23}$ The bright, dark, and dim areas are due to the difference of electron density and electric field in these areas. When the pulse has $2 \mu \mathrm{s}$ break as shown in the top panel in Fig. 4, species produced by the fall-phase discharge may not completely recombine before the onset of the subsequent discharge at the voltage rise phase. Therefore, because of the short pause between the two discharges, the density of residual electrons produced during the preceding fall phase may remain high before the next discharge is ignited at the rising edge of the voltage pulse. The length of the plasma plume during the fall phase appears to be much shorter compared to the rise phase. Meanwhile, this length is the same as the length of the bright area I. Hence, the residual electron density within area I is relatively high because of the discharge during the falling edge of the voltage pulse. Consequently, area I becomes conductive at the onset of the rise phase. However, its conductivity is not too high, thus a relatively high electric field within area I is still needed to sustain a certain degree of ionization and excitation. This is why the plasma of area I remains stable for a relatively long time. It also needs to be emphasized that Sec. I is not an accumulation of plasma bullets. On the other hand, for area II in the top panel of Fig. 4, it is similar to the traditional plasma bullet behavior. Indeed, as the discharge during the falling edge cannot reach this area, the dim zone is due to the propagation of the plasma bullet.

For the bottom image in Fig. 4, as the time between the discharge at the falling edge of the voltage pulse and the discharge at the rising edge of the subsequent pulse is short, the residual electron density in front of the nozzle is even higher when the discharge is ignited during the rising edge. Hence, area I in Fig. 4 (bottom panel) has an even higher electric conductivity and a weaker electric field. The weaker electric field results in a low electron temperature, so the electrons cannot gain enough energy to ionize or excite atoms or molecules. That is the reason why this area appears dark.

However, it still remains unclear in which specific ranges of the electron density the plasma plume appears dark or bright. To have conclusive answers to these questions, computer simulations are needed to better understand the relationships among the optical emission spectra of the discharge, electron density, and the electric field distributions along the plasma plume.

For the snake-like propagation behavior depicted in Fig. $5,{ }^{27}$ the exact reasons also still remain unclear. One of the possible reasons is due to the turbulence flow of the working gas. In this case, the densities of long-lifetime charged species are not uniform within the tube and on the inner surface of the tube just before the next voltage pulse. When the discharge is initiated, the plasma plume propagates towards the location where (1) the electric field is high (part of it is due to the non-uniform distribution of surface charges) and (2) the concentration of background reactive species (such as charged particles) is high. These factors contribute to the observed snake-like propagation behavior.
However, it is still not clear whether these observations are only due to nonuniform distributions of charged electrons or ion, or also some other species, for example, excited species in charge-neutral states. Because of the small sizes and fast dynamic nature of the streamer propagation, higher spatial and temporal resolutions are needed, which still remains very challenging from the experimental measurements and equipment cost points of view. Thus, computer simulations will continue to be a very useful tool to describe and quantify the numerous experimental observations.

\section{CONCLUSION}

In this review, we have discussed one of the most intriguing features of the atmospheric pressure plasma jets, namely, their regular, repeatable behavior. This type of plasma streamer discharges features plasma bullets which propagate in the same direction of the gas flow and reach predictable positions depending on the characteristics of applied voltage and nature and pressure of working gas used. This regular, quasi-deterministic behavior is drastically different from the random, stochastic behavior of traditional types of plasma streamer discharges. The ability to control plasma streamer propagation is appealing for several practical applications including but not limited to fabrication and processing of advanced functional materials, biotechnology, and biomedical treatments.

We have examined some of the key physical aspects of repeatability of the guided ionization waves which still remain largely unclear despite more than a decade of intense research efforts worldwide. These international efforts have largely focused on better understanding of the propagation dynamics of the plasma bullets and other traditional types of plasma streamer discharges. The physical mechanisms leading to the vastly distinctive features of propagation and other characteristics of the guided plasma streamers are related to the effects of electric charges within and around the channel where the plasma jets propagate.

One particularly important conclusion suggests that highly reproducible, regular behavior of the plasma bullets owes the sufficiently high density of electrons at the discharge initiation stage within the channel. These electrons are referred to as seed electrons. The condition that the density of seed electrons should be sufficiently high, namely, of the order of $\sim 10^{9} \mathrm{~cm}^{-3}$ justifies the introduction of the special interpretative term related to this kind of discharges, namely, high seed electron density (HSED) plasma discharges. This particular criterion and other considerations made it possible to distinguish guided plasma streamers from traditional positive streamer discharges. We articulate that the high-concentration of seed electrons determines the threshold when atmospheric-pressure plasma plumes show a distinctive repeatable propagation mode.

Critical review of the vast amount of available literature confirms that common positive streamer discharges are sustained under conditions when the density of initial electrons created by photoionization or other means is below the threshold stipulated by the HSED criterion. When so happens, the streamer propagation mode is random (also referred 
to as stochastic) not only in terms of propagation direction and distance travelled but also in terms of the dynamics of streamer development and propagation. The examination of the results published so far makes us confident to state that the HSED criterion can be used as the valid first approximation to predict and describe the transition from the irregular streamers to highly repeatable plasma bullet propagation.

We also emphasize that if effective means to adjust the seed electron density in the vicinity of the threshold described by the HSED criterion could be developed, this would potentially lead to step changes in our ability to control the behavior of atmospheric-pressure plasma jets in diverse applications including plasma health care and medicine, industrial biotechnology, food and agribusiness, environmental monitoring and remediation, fabrication of advanced materials and devices using nanotechnology, and several others.

We would like to conclude this review with a philosophical remark using a common proverb "Every seed knows its time." We hope that our effort will help eventually to determine the moment for every plasma bullet to emerge, and how many seed electrons do we need to "plant" in the propagation channel to make this happen.

Finally, we would like to acknowledge the many valuable contributions of the very large number of researchers working in this and relevant areas and apologize that some of these contributions have not been included in this review. We hope that our effort will stimulate discussions and collaborations in this topical area of research and will appreciate and further comments and suggestions for future collaborative research and creation of common information exchange platforms.

\section{ACKNOWLEDGMENTS}

This work was partially supported by the National Natural Science Foundation of China (Grant Nos. 51277087, 51477066, and 51625701). K.O. thanks the Australian Research Council for partial support.

\section{${ }^{1}$ M. Laroussi, IEEE Trans. Plasma Sci. 24, 1188 (1996).}

${ }^{2}$ A. Kramer, J. Lademann, C. Bender, A. Sckell, B. Hartmann, S. Münch, P. Hinz, A. Ekkernkamp, R. Matthes, I. Koban, I. Partecke, C. D. Heidecke, K. Masur, S. Reuter, K. D. Weltmann, S. Koch, and O. Assadian, Clin. Plasma Med. 1, 11 (2013).

${ }^{3}$ A. Fridman and G. Friedman, Plasma Medicine (Wiley, New York, 2013).

${ }^{4}$ T. von Woedtke, S. Reuter, K. Masur, and K.-D. Weltmann, Phys. Rep. 530, 291 (2013).

${ }^{5}$ D. B. Graves, Phys. Plasmas 21, 080901 (2014).

${ }^{6}$ A. Shashurin, M. Keidar, S. Bronnikov, R. A. Jurjus, and M. A. Stepp, Appl. Phys. Lett. 93, 181501 (2008).

${ }^{7}$ Y. Li, M. Kang, H. Uhm, G. Lee, E. Choi, and I. Han, Sci. Rep. 7, 45781 (2017).

${ }^{8}$ N. Y. Babaeva and M. J. Kushner, J. Phys. D: Appl. Phys. 46, 025401 (2013).

${ }^{9}$ M. Ishaq, M. M. Evans, and K. Ostrikov, Int. J. Cancer 134, 1517 (2014).

${ }^{10}$ M. Yousfi, N. Merbahi, A. Pathak, and O. Eichwald, Fundam. Clin. Pharmacol. 28, 123 (2014).

${ }^{11}$ Z. Fang, T. Shao, J. Yang, and C. Zhang, Eur. Phys. J. D 70, 3 (2016).

${ }^{12}$ X. Lu, G. V. Naidis, M. Laroussi, and K. Ostrikov, Phys. Rep. 540, 123 (2014).

${ }^{13}$ Y. Xia, W. Wang, D. Liu, Y. Peng, Y. Song, L. Ji, Y. Zhao, Z. Qi, X. Wang, and B. Li, Plasma Processes Polym. 14, e1600132 (2017).
${ }^{14}$ S. Kos, T. Blagus, M. Cemazar, G. Filipic, G. Sersa, and U. Cvelbar, PLoS One 12, e0174966 (2017).

${ }^{15}$ A. Shashurin, M. N. Shneider, A. Dogariu, R. B. Miles, and M. Keidar, Appl. Phys. Lett. 94, 231504 (2009).

${ }^{16}$ M. Laroussi and X. Lu, Appl. Phys. Lett. 87, 113902 (2005).

${ }^{17}$ C. Zhang, T. Shao, R. Wang, Z. Zhou, Y. Zhou, and P. Yan, Phys. Plasmas 21, 103505 (2014).

${ }^{18}$ X. Lu and M. Laroussi, J. Appl. Phys. 100, 063302 (2006).

${ }^{19}$ X. Zhang, D. Liu, R. Zhou, Y. Song, Y. Sun, Q. Zhang, J. Niu, H. Fan, and S. Yang, App. Phys. Lett. 104, 043702 (2014).

${ }^{20}$ J. Kolb, A. Mohamed, R. Price, R. Swanson, A. Bowman, R. Chiavarini, M. Stacey, and K. Schoenbach, Appl. Phys. Lett. 92, 241501 (2008).

${ }^{21}$ S. Nijdam, G. Wormeester, E. Veldhuizen, and U. Ebert, J. Phys. D: Appl. Phys. 44, 455201 (2011).

${ }^{22}$ X. Lu, Z. Jiang, Q. Xiong, Z. Tang, X. Hu, and Y. Pan, Appl. Phys. Lett. 92, 081502 (2008).

${ }^{23}$ Y. Xian, P. Zhang, X. Lu, X. Pei, S. Wu, Q. Xiong, and K. Ostrikov, Sci. Rep. 3, 1599 (2013).

${ }^{24}$ W. Yi and P. Williams, J. Phys. D: Appl. Phys. 35, 205 (2002).

${ }^{25}$ R. Zeng and S. Chen, J. Phys. D: Appl. Phys. 46, 485201 (2013).

${ }^{26}$ S. Park, U. Cvelbar, W. Choe, and S. Y. Moon, Nat. Commun. 9, 371 (2018).

${ }^{27}$ S. Wu, Z. Wang, Q. Huang, X. Tan, X. Lu, and K. Ostrikov, Phys. Plasmas 20, 023503 (2013).

${ }^{28}$ J. Townsend, Nature 62, 340 (1900).

${ }^{29}$ Y. P. Raizer, Gas Discharge Physics (Springer, Berlin, 1991).

${ }^{30} \mathrm{~L}$. Loeb, Fundamental Processes of Electrical Discharge in Gases (Wiley, New York, 1939).

${ }^{31}$ L. Loeb, B. Leonard, and A. Kip, J. Appl. Phys. 10, 142 (1939).

${ }^{32}$ H. Raether, Z. Phys. 112, 464 (1939).

${ }^{33}$ J. Meek, Phys. Rev. 57, 722 (1940).

${ }^{34}$ P. Bruggeman and R. Brandenburg, J. Phys. D: Appl. Phys. 46, 464001 (2013).

${ }^{35}$ N. Yu. Babaeva, G. V. Naidis, and M. J. Kushner, Plasma Sources Sci. Technol. 27, 015016 (2018).

${ }^{36}$ H. Höft, M. Kettlitz, M. M. Becker, T. Hoder, D. Loffhagen, R. Brandenburg, and K.-D. Weltmann, J. Phys. D: Appl. Phys. 47, 465206 (2014).

${ }^{37}$ Y. Inada, K. Aono, R. Ono, A. Kumada, K. Hidaka, and M. Maeyama, J. Phys. D: Appl. Phys. 50, 174005 (2017).

${ }^{38}$ M. Okubo, Phys. Plasmas 22, 123515 (2015).

${ }^{39}$ M. Bujotzek, M. Seeger, F. Schmidt, M. Koch, and C. Franck, J. Phys. D: Appl. Phys. 48, 245201 (2015).

${ }^{40}$ C. Köhn, O. Chanrion, and T. Neubert, Plasma Sources Sci. Technol. 26, 015006 (2017).

${ }^{41}$ A. Luque and U. Ebert, New J. Phys. 16, 013039 (2014).

${ }^{42}$ M. Šimek, B. Pongrác, V. Babický, M. Člupek, and P. Lukeš, Plasma Sources Sci. Technol. 26, 07LT01 (2017).

${ }^{43}$ A. H. Markosyan, S. Dujko, and U. Ebert, J. Phys. D: Appl. Phys. 46, 475203 (2013).

${ }^{44}$ X. Pei, S. Wu, Y. Xian, X. Lu, and Y. Pan, IEEE Trans. Plasma Sci. 42, 1206-1210 (2014).

${ }^{45}$ X. Pei, J. Liu, Y. Xian, and X. Lu, J. Phys. D: Appl. Phys. 47, 145204 (2014).

${ }^{46}$ F. Tholin and A. Bourdon, Plasma Phys. Controlled Fusion 57, 014016 (2015).

${ }^{47}$ J. Zhang, Y. Wang, and D. Wang, IEEE Trans. Plasma Sci. 46, 19 (2018).

${ }^{48}$ J. Stephens, A. Fierro, J. Dickens, and A. Neuber, J. Phys. D: Appl. Phys. 47, 325501 (2014).

${ }^{49}$ F. Taccogna and F. Pellegrini, J. Phys. D: Appl. Phys. 51, 064001 (2018).

${ }^{50}$ S. Nijdam, E. Takahashi, A. H. Markosyan, and U. Ebert, Plasma Sources Sci. Technol. 23, 025008 (2014).

${ }^{51}$ G. V. Naidis, Plasma Processes Polym. 14, 1600127 (2017).

${ }^{52}$ A. Komuro, R. Ono, and T. Oda, J. Phys. D: Appl. Phys. 45, 265201 (2012).

${ }^{53}$ J.-M. Plewa, O. Eichwald, O. Ducasse, P. Dessante, C. Jacobs, N. Renon, and M. Yousfi, J. Phys. D: Appl. Phys. 51, 095206 (2018).

${ }^{54}$ T. Houba and S. Roy, J. Appl. Phys. 118, 233303 (2015).

${ }^{55}$ C. D. Pintassilgo and V. Guerra, Plasma Sources Sci. Technol. 24, 055009 (2015).

${ }^{56}$ P. Suanpoot, G. Han, J. Sornsakdanuphap, H. Uhm, G. Cho, and E. Choi, IEEE Trans. Plasma Sci. 43, 2207 (2015).

${ }^{57}$ A. Wagner, D. Mariotti, K. Yurchenko, and T. Das, Phys. Rev. E 80, 065401 (2009). 
${ }^{58}$ S. Pancheshnyi, Plasma Sources Sci. Technol. 24, 015023 (2015).

${ }^{59}$ M. Jiang, Y. Li, H. Wang, P. Zhong, and C. Liu, Phys. Plasmas 25, 012127 (2018).

${ }^{60}$ M. Janda, Z. Machala, L. Dvonč, D. Lacoste, and C. O. Laux, J. Phys. D: Appl. Phys. 48, 035201 (2015).

${ }^{61}$ S.-Y. Yoon, G.-H. Kim, S.-J. Kim, B. Bae, N.-K. Kim, H. Lee, N. Bae, S. Ryu, S. J. Yoo, and S. B. Kim, Phys. Plasmas 24, 013513 (2017).

${ }^{62}$ F. Tholin and A. Bourdon, J. Phys. D: Appl. Phys. 46, 365205 (2013).

${ }^{63}$ E. Takahashi, S. Kato, H. Furutani, A. Sasaki, Y. Kishimoto, K. Takada, S. Matsumura, and H. Sasaki, J. Phys. D: Appl. Phys. 44, 302001 (2011).

${ }^{64}$ M. Šimek, P. F. Ambrico, and V. Prukner, J. Phys. D: Appl. Phys. 50, 504002 (2017).

${ }^{65}$ S. Nijdam, E. Takahashi, J. Teunissen, and U. Ebert, New J. Phys. 16, 103038 (2014).

${ }^{66}$ T. Huiskamp, A. J. M. Pemen, W. F. L. M. Hoeben, F. J. C. M. Beckers, and E. J. M. van Heesch, J. Phys. D: Appl. Phys. 46, 165202 (2013).

${ }^{67}$ X. Wen, Q. Li, J. Li, and C. Ren, Plasma Sci. Technol. 19, 085401 (2017).

${ }^{68}$ H. Höft, M. Kettlitz, T. Hoder, K.-D. Weltmann, and R. Brandenburg, J. Phys. D: Appl. Phys. 46, 095202 (2013).

${ }^{69}$ M. Janda, Z. Machala, A. Niklová, and V. Martĩsoviť, Plasma Sources Sci. Technol. 21, 045006 (2012).

${ }^{70}$ D. A. Xu, M. N. Shneider, D. A. Lacoste, and C. O. Laux, J. Phys. D: Appl. Phys. 47, 235202 (2014).

${ }^{71}$ D. Trienekens, J. Stephens, A. Fierro, J. Dickens, and A. Neuber, J. Phys. D: Appl. Phys. 49, 035201 (2016).

${ }^{72}$ Y. Zhang, H.-Y. Wang, W. Jiang, and A. Bogaerts, New J. Phys. 17, 083056 (2015)

${ }^{73}$ A. Komuro and R. Ono, J. Phys. D: Appl. Phys. 47, 155202 (2014).

${ }^{74}$ S. Lanier, I. Shkurenkov, I. V. Adamovich, and W. R. Lempert, Plasma Sources Sci. Technol. 24, 025005 (2015).

${ }^{75}$ D. L. Rusterholtz, D. A. Lacoste, G. D. Stancu, D. Z. Pai, and C. O. Laux, J. Phys. D: Appl. Phys. 46, 464010 (2013).

${ }^{76}$ M. Jiang, Y. Li, H. Wang, and C. Liu, Phys. Plasmas 24, 102112 (2017).

${ }^{77}$ A. Komuro, K. Takahashi, and A. Ando, Plasma Sources Sci. Technol. 24, 055020 (2015).

${ }^{78}$ G. A. Mesyats and M. I. Yalandin, IEEE Trans. Plasma Sci. 37, 785 (2009).

${ }^{79}$ O. Chanrion and T. Neubert, J. Geophys. Res. 115, A00E32, https:// doi.org/10.1029/2009JA014774 (2010).

${ }^{80} \mathrm{U}$. Ebert et al., Nonlinearity 24, C1 (2011).

${ }^{81}$ A. Luque and U. Ebert, J. Comput. Phys. 231, 904 (2012).

${ }^{82}$ C. Li, J. Teunissen, M. Nool, W. Hundsdorfer, and U. Ebert, Plasma Sources Sci. Technol. 21, 055019 (2012).

${ }^{83}$ N. Y. Babaeva and G. Naidis, J. Phys. D: Appl. Phys. 29, 2423 (1996).

${ }^{84}$ N. Y. Babaeva and G. Naidis, IEEE Trans. Plasma Sci. 25, 375 (1997).

${ }^{85}$ G. Wormeester, S. Nijdam, and U. Ebert, Jpn. J. Appl. Phys., Part 1 50, 08JA01 (2011)

${ }^{86}$ A. Luque and U. Ebert, Phys. Rev. E 84, 046411 (2011).

${ }^{87}$ E. Takahashi, S. Kato, A. Sasaki, Y. Kishimoto, and H. Furutani, J. Phys. D: Appl. Phys. 44, 075204 (2011).

${ }^{88}$ D. Mathew, H. M. J. Bastiaens, K. J. Boller, and P. J. M. Peters, J. Appl. Phys. 102, 033305 (2007).

${ }^{89}$ S. Dujko, A. H. Markosyan, R. D. White, and U. Ebert, J. Phys. D: Appl. Phys. 46, 475202 (2013).

${ }^{90}$ R. Ono, Y. Teramoto, and T. Oda, J. Phys. D: Appl. Phys. 43, 345203 (2010).

${ }^{91}$ Z. Xiong and M. J. Kushner, Plasma Sources Sci. Technol. 23, 065041 (2014).

${ }^{92}$ S. K. Dhali and P. F. Williams, J. Appl. Phys. 62, 4696 (1987).

${ }^{93}$ P. A. Vitello, B. M. Penetrante, and J. N. Bardsley, Phys. Rev. E 49, 5574 (1994)

${ }^{94}$ A. Bourdon, Z. Bonaventura, and S. Celestin, Plasma Sources Sci. Technol. 19, 034012 (2010).

${ }^{95}$ A. Luque, V. Ratushnaya, and U. Ebert, J. Phys. D: Appl. Phys. 41, 234005 (2008).

${ }^{96}$ A. M. Cravath, Phys. Rev. 33, 605 (1929).

${ }^{97}$ T. H. Teich, Z. Phys. 199, 378 (1967)

${ }^{98}$ T. H. Teich, Z. Phys. 199, 395 (1967).

${ }^{99}$ L. B. Loeb, Rev. Mod. Phys. 8, 267 (1936).

${ }^{100}$ H. Raether, Z. Phys. 110, 611 (1938).

${ }^{101}$ A. Przybylski, Z. Phys. 151, 264 (1958).

${ }^{102}$ G. W. Penney and G. T. Hummert, J. Appl. Phys. 41, 572 (1970).

${ }^{103}$ N. Liu and V. Pasko, J. Phys. D: Appl. Phys. 39, 327 (2006).
${ }^{104}$ A. Sun, J. Teunissen, and U. Ebert, J. Phys. D: Appl. Phys. 47, 445205 (2014).

${ }^{105}$ G. Naidis, Tech. Phys. Lett. 23, 493 (1997).

${ }^{106}$ N. Liu, S. Célestin, A. Bourdon, V. P. Pasko, P. Ségur, and E. Marode, Appl. Phys. Lett. 91, 211501 (2007).

${ }^{107}$ G. Naidis, Plasma Sources Sci. Technol. 15, 253 (2006).

${ }^{108}$ T. Briels, E. M. van Veldhuizen, and U. Ebert, IEEE Trans. Plasma Sci. 33, 264 (2005).

${ }^{109}$ P. Segur, A. Bourdon, E. Marode, D. Bessieres, and J. Paillol, Plasma Sources Sci. Technol. 15, 648 (2006).

${ }^{110}$ E. Gerken, U. Inan, and C. Barrington-Leigh, Geophys. Res. Lett. 27, 2637, https://doi.org/10.1029/2000GL000035 (2000).

${ }^{111}$ N. Liu and V. Pasko, J. Phys. D: Appl. Phys. 43, 082001 (2010).

${ }^{112}$ N. Liu and V. Pasko, J. Geophys. Res. 109, A09306, https://doi.org/ 10.1029/2004JA010692 (2004)

${ }^{113}$ S. Pancheshnyi, Plasma Sources Sci. Technol. 14, 645 (2005).

${ }^{114}$ M. B. Zhelezniak, A. Kh. Mnatsakanian, and S. V. Sizykh, High Temp. 20, 357 (1982).

${ }^{115}$ A. Luque, U. Ebert, C. Nontijn, and W. Hundsdorfer, Appl. Phys. Lett. 90, 081501 (2007).

${ }^{116}$ A. Bourdon, V. Pasko, N. Liu, S. Celestin, P. Segur, and E. Marode, Plasma Sources Sci. Technol. 16, 656 (2007).

${ }^{117}$ G. Wormeester, S. Pancheshnyi, A. Luque, S. Nijdam, and U. Ebert, J. Phys. D: Appl. Phys. 43, 505201 (2010).

${ }^{118}$ S. Nijdam, F. M. J. H. van de Wetering, R. Blanc, E. M. van Veldhuizen, and U. Ebert, J. Phys. D: Appl. Phys. 43, 145204 (2010).

${ }^{119}$ S. Pancheshnyi, S. M. Starikovskaia, and A. Yu. Starikovskii, J. Phys. D: Appl. Phys. 34, 105 (2001).

${ }^{120}$ A. Luque, U. Ebert, and W. Hundsdorfer, Phys. Rev. Lett. 101, 075005 (2008).

${ }^{121}$ S. V. Pancheshnyi, M. Nudnova, and A. Y. Starikovskii, Phys. Rev. E 71, 016407 (2005)

${ }^{122}$ T. Clevis, S. Nijdam, and U. Ebert, J. Phys. D: Appl. Phys. 46, 045202 (2013).

${ }^{123}$ S. Chen, L. C. J. Heijmans, R. Zeng, S. Nijdam, and U. Ebert, J. Phys. D: Appl. Phys. 48, 175201 (2015).

${ }^{124}$ C. Douat, G. Bauville, M. Fleury, M. Laroussi, and V. Puech, Plasma Sources Sci. Technol. 21, 034010 (2012).

${ }^{125}$ Q. Xiong, X. P. Lu, K. Ostrikov, Y. Xian, C. Zou, Z. Xiong, and Y. Pan, Phys. Plasmas 17, 043506 (2010).

${ }^{126}$ N. Mericam-Bourdet, M. Laroussi, A. Begum, and E. Karakas, J. Phys. D: Appl. Phys. 42, 055207 (2009).

${ }^{127}$ K. Urabe, Y. Ito, K. Tachibana, and B. N. Ganguly, Appl. Phys. Express 1, 066004 (2008).

${ }^{128}$ J. L. Walsh and M. G. Kong, Appl. Phys. Lett. 91, 221502 (2007).

${ }^{129}$ Y. Xian, X. Lu, Y. Cao, P. Yang, Q. Xiong, Z. Jiang et al., IEEE Trans. Plasma Sci. 37, 2068 (2009).

${ }^{130}$ G. B. Sretenović, O. Guaitella, A. Sobota, I. B. Krstić, V. V. Kovačević, B. M. Obradović, and M. M. Kuraica, J. Appl. Phys. 121, 123304 (2017).

${ }^{131}$ J. L. Walsh, F. Iza, N. B. Janson, V. J. Law, and M. G. Kong, J. Phys. D: Appl. Phys. 43, 075201 (2010).

${ }^{132}$ X. Lu, Z. Jiang, Q. Xiong, Z. Tang, Z. Xiong, J. Hu et al., IEEE Trans. Plasma Sci. 36, 988 (2008).

${ }^{133}$ S. Wu, Q. Huang, Z. Wang, and X. Lu, IEEE Trans. Plasma Sci. 39, 2286 (2011).

${ }^{134}$ M. Laroussi, Plasma Processes Polym. 2, 391 (2005).

${ }^{135}$ Plasma Medicine: Applications of Low Temperature Gas Plasmas in Medicine and Biology, edited by M. Laroussi, M. Kong, G. Morfill, and W. Stolz (Cambridge University Press, Cambridge, 2012).

${ }^{136}$ M. Keidar, A. Shashurin, O. Volotskova, M. Ann Stepp, P. Srinivasan, A. Sandler et al., Phys. Plasmas. 20, 057101 (2013).

${ }^{137}$ O. Goossens, E. Dekempeneer, D. Vangeneugden, R. Van de Leest, and C. Leys, Surf. Coat. Technol. 142-144, 474 (2001).

${ }^{138}$ K. Bazaka, M. V. Jacob, W. Chrzanowski, and K. Ostrikov, RSC Adv. 5, 48739 (2015)

${ }^{139}$ J. Winter, K. Wende, K. Masur, S. Iseni, M. Dunnbier, M. U. Hammer, H. Tresp, K. D. Weltmann, and S. Reuter, J. Phys. D: Appl. Phys. 46, 295401 (2013).

${ }^{140} \mathrm{P}$. Viegas, F. Péchereau, and A. Bourdon, Plasma Sources Sci. Technol. 27, 025007 (2018).

${ }^{141}$ T. Murakami, K. Niemi, T. Gans, D. O'Connell, and W. G. Graham, Plasma Sources Sci. Technol. 22, 015003 (2013)

${ }^{142}$ W. van Gaens and A. Bogaerts, J. Phys. D: Appl. Phys. 46, 275201 (2013). 
${ }^{143}$ G. Y. Park, Y. J. Hong, H. W. Lee, J. Y. Sim, and J. K. Lee, Plasma Processes Polym. 7, 281 (2010).

${ }^{144}$ T. Murakami, K. Niemi, T. Gans, D. O'Connell, and W. G. Graham, Plasma Sources Sci. Technol. 23, 025005 (2014).

${ }^{145}$ K. Niemi, J. Waskoenig, N. Sadeghi, T. Gans, and D. O'Connell, Plasma Sources Sci. Technol. 20, 055005 (2011).

${ }^{146}$ A. Schmidt-Bleker, J. Winter, S. Iseni, M. Dünnbier, K.-D. Weltmann, and S. Reuter, J. Phys. D: Appl. Phys. 47, 145201 (2014).

${ }^{147}$ G. V. Naidis, Plasma Sources Sci. Technol. 22, 035015 (2013).

${ }^{148}$ W. van Gaens and A. Bogaerts, Plasma Sources Sci. Technol. 23, 035015 (2014).

${ }^{149}$ W. van Gaens, P. J. Bruggeman, and A. Bogaerts, New J. Phys. 16, 063054 (2014)

${ }^{150}$ W. van Gaens, S. Iseni, A. Schmidt-Bleker, K.-D. Weltmann, S. Reuter, and A. Bogaerts, New J. Phys. 17, 033003 (2015).

${ }^{151}$ G. V. Naidis, Plasma Sources Sci. Technol. 23, 065014 (2014).

${ }^{152}$ B. Niermann, T. Hemke, N. Y. Babaeva, M. Böke, M. J. Kushner, T. Mussenbrock et al., J. Phys. D: Appl. Phys. 44, 485204 (2011).

${ }^{153}$ S. Schneider, J. W. Lackmann, F. Narberhaus, J. E. Bandow, B. Denis, and J. Benedikt, J. Phys. D: Appl. Phys. 44, 295201 (2011).

${ }^{154}$ D. Breden and L. L. Raja, Plasma Sources Sci. Technol. 23, 065020 (2014).

${ }^{155}$ X. Liu, X. Pei, X. Lu, and D. Liu, Plasma Sources Sci. Technol. 23, 035007 (2014)

${ }^{156}$ N. Y. Babaeva and M. J. Kushner, Plasma Sources Sci. Technol. 23, 015007 (2014)

${ }^{157}$ S. A. Norberg, E. Johnsen, and M. J. Kushner, Plasma Sources Sci. Technol. 24, 035026 (2015).

${ }^{158}$ F. Iza, G. J. Kim, S. M. Lee, J. K. Lee, J. L. Walsh, Y. T. Zhang, and M. G. Kong, Plasma Processes Polym. 5, 322 (2008).

${ }^{159}$ G. Dilecce, Plasma Sources Sci. Technol. 23, 015011 (2014).

${ }^{160} \mathrm{X}$. Lu, G. V. Naidis, M. Laroussi, S. Reuter, D. B. Graves, and K. Ostrikov, Phys. Rep. 630, 1 (2016).

${ }^{161}$ S. Reuter, J. Winter, S. Iseni, S. Peters, A. Schmidt-Bleker, M. Dünnbier, J. Schäfer, R. Foest, and K.-D. Weltmann, Plasma Sources Sci. Technol. 21, 034015 (2012).

${ }^{162}$ S. Reuter, A. Schmidt-Bleker, S. Iseni, J. Winter, and K.-D. Weltmann, IEEE Trans. Plasma Sci. 42, 2428 (2014).

${ }^{163}$ G. Dilecce, L. M. Martini, P. Tosi, M. Scotoni, and S. D. Benedictis, Plasma Sources Sci. Technol. 24, 034007 (2015).

${ }^{164}$ S. Iseni, A. Schmidt-Bleker, J. Winter, K.-D. Weltmann, and S. Reuter, J. Phys. D: Appl. Phys. 47, 152001 (2014).

${ }^{165}$ D. Levko, M. Pachuilo, and L. L. Raja, J. Phys. D: Appl. Phys. 50, 354004 (2017).

${ }^{166}$ M. I. Hasan, U. Cvelbar, J. W. Bradley, and J. L. Walsh, J. Phys. D: Appl. Phys. 50, 205201 (2017).

${ }^{167}$ D. Riès, G. Dilecce, E. Robert, P. F. Ambrico, S. Dozias, and J. M. Pouvesle, J. Phys. D: Appl. Phys. 47, 275401 (2014).

${ }^{168}$ E. Robert, V. Sarron, T. Darny, D. Riès, S. Dozias, J. Fontane, L. Joly, and J.-M. Pouvesle, Plasma Sources Sci. Technol. 23, 012003 (2014).

${ }^{169}$ T. Shao, C. Zhang, R. Wang, Y. Zhou, Q. Xie, and Z. Fang, IEEE Trans. Plasma Sci. 43, 726 (2015).

${ }^{170}$ C. Zhang, T. Shao, R. Wang, W. Huang, Z. Niu, and E. Schamiloglu, IEEE Trans. Dielectr. Electr. Insul. 22, 1907 (2015).
${ }^{171}$ S. Wu, X. Lu, and Y. Pan, Curr. Appl. Phys. 13, S1 (2013).

${ }^{172}$ L. Nie, L. Chang, Y. Xian, and X. Lu, Phys. Plasmas 23, 093518 (2016).

${ }^{173}$ S. Wu and X. Lu, Phys. Plasmas 21, 123509 (2014).

${ }^{174}$ S. Wu, X. Lu, D. Liu, Y. Yang, Y. Pan, and K. Ostrikov, Phys. Plasmas 21, 103508 (2014)

${ }^{175}$ L. Nie, Y. Xian, X. Lu, and K. Ostrikov, Phys. Plasmas 24, 043502 (2017).

${ }^{176}$ A. V. Phelps, J. Res. Natl. Inst. Stand. Technol. 95, 407 (1990).

${ }^{177}$ H. B. Pedersen et al., Phys. Rev. A: At., Mol., Opt. Phys. 72, 012712 (2005).

${ }^{178}$ Y. S. Cao and R. Johnsen, J. Chem. Phys. 95, 7356 (1991).

${ }^{179}$ D. X. Liu, M. Z. Rong, X. H. Wang, F. Iza, M. G. Kong, and P. Bruggeman, Plasma Processes Polym. 7, 846 (2010).

${ }^{180}$ B. M. Penetrante et al., Plasma Sources Sci. Technol. 6, 251 (1997).

${ }^{181}$ J. Dutton, J. Phys. Chem. Ref. Data 4, 577 (1975).

${ }^{182}$ D. Breden, K. Miki, and L. L. Raja, Plasma Sources Sci. Technol. 21, 034011 (2012).

${ }^{183}$ A. Shashurin, M. N. Shneider, and M. Keidar, Plasma Sources Sci. Technol. 21, 049601 (2012).

${ }^{184}$ A. Begum, M. Laroussi, and M. R. Pervez, AIP Adv. 3, 062117 (2013).

${ }^{185}$ L. Chang, L. Nie, Y. Xian, and X. Lu, Phys. Plasmas 23, 123513 (2016).

${ }^{186}$ D. Breden, K. Miki, and L. Raja, Appl. Phys. Lett. 99, 111501 (2011).

${ }^{187}$ Z. Xiong and M. J. Kushner, Plasma Sources Sci. Technol. 21, 034001 (2012).

${ }^{188}$ J. Boeuf, L. Yang, and L. Pitchford, J. Phys. D: Appl. Phys. 46, 015201 (2013).

${ }^{189}$ Z. Xiong, E. Robert, V. Sarron, J. Pouvesle, and M. J. Kushner, J. Phys. D: Appl. Phys. 45, 275201 (2012).

${ }^{190}$ G. V. Naidis, J. Phys. D: Appl. Phys. 43, 402001 (2010).

${ }^{191}$ G. V. Naidis, J. Phys. D: Appl. Phys. 44, 215203 (2011).

${ }^{192}$ G. V. Naidis, Appl. Phys. Lett. 98, 141501 (2011).

${ }^{193}$ G. V. Naidis, J. Appl. Phys. 112, 103304 (2012).

${ }^{194}$ M. Yousfi, O. Eichwald, N. Merbahi, and N. Jomma, Plasma Sources Sci. Technol. 21, 045003 (2012).

${ }^{195}$ Y. Sakiyama, D. Graves, J. Jarrige, and M. Laroussi, Appl. Phys. Lett. 96, 041501 (2010)

${ }^{196}$ F. Liu, D. Zhang, and D. Wang, Thin Solid Films 521, 261 (2012).

${ }^{197}$ G. V. Naidis, Plasma Sources Sci. Technol. 21, 034003 (2012).

${ }^{198}$ G. V. Naidis and J. L. Walsh, J. Phys. D: Appl. Phys. 46, 095203 (2013).

${ }^{199}$ K. H. Becker, K. H. Schoenbach, and J. G. Eden, J. Phys. D: Appl. Phys. 39, R55 (2006).

${ }^{200}$ S.-J. Park, J. G. Eden, and K.-H. Park, Appl. Phys. Lett. 84, 4481 (2004).

${ }^{201}$ T. Murakami, K. Niemi, T. Gans, D. O. Connell, and W. G. Graham, Plasma Sources Sci. Technol. 22, 015003 (2013).

${ }^{202}$ N. Aleksandrov and E. Anokhin, J. Phys. B: At., Mol., Opt. Phys. 44, 115202 (2011).

${ }^{203}$ A. Mnatsakanyan and G. Naidis, in Reviews of Plasma Chemistry, edited by B. Smirnov (Consultants Bureau, New York, 1991), Vol. 1, p. 259.

${ }^{204}$ D. W. Goodson, R. J. Corbin, and L. Frommhold, Phys. Rev. A 9, 2049 (1974).

${ }^{205}$ B. C. O’Neil and J. D. Craggs, J. Phys. B: At., Mol., Opt. Phys. 6, 2625 (1973). 